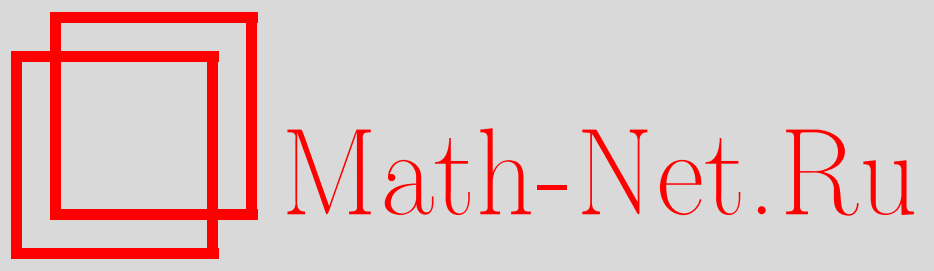

Ю. Э. Андерсен, Л. О. Чехов, П. Норбари, Р. К. Пеннер, Топологическая рекурсия для гауссовых средних и когомологические теории поля, ТМФ, 2015, том 185, номер 3, 371-409

DOI: https://doi.org/10.4213/tmf8951

Использование Общероссийского математического портала Math-Net.Ru подразумевает, что вы прочитали и согласны с пользовательским соглашением http://www.mathnet.ru/rus/agreement

Параметры загрузки:

IP : 44.207 .124 .84

26 апреля 2023 г., 16:05:02

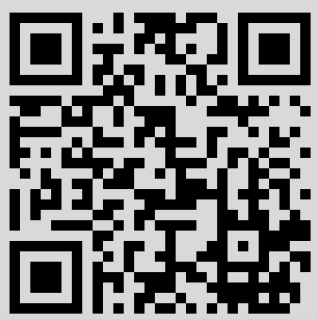




\title{
ТОПОЛОГИЧЕСКАЯ РЕКУРСИЯ ДЛЯ ГАУССОВЫХ СРЕДНИХ И КОГОМОЛОГИЧЕСКИЕ ТЕОРИИ ПОЛЯ
}

\begin{abstract}
Приводятся явные соотношения между разложенными по родам $s$-петлевыми средними гауссовой матричной модели и членами разложения по родам матричной модели Концевича-Пеннера, которая задает производящую функцию для объемов дискретизованных (открытых) пространств модулей $M_{g, s}^{\text {disc }}$ (дискретных объемов). С помощью этих соотношений гауссовы средние во всех порядках разложения по родам представляются в виде многочленов от специальных переменных времен с коэффициентами, оказывающимися инвариантными интегралами потомков для подлежащей когомологической теории поля. Топологическая рекурсия для гауссовой модели переводится в рекуррентные соотношения на коэффициенты этих многочленов, что позволяет доказать их целочисленность и положительность. Найдены коэффициенты в первом поправочном порядке для $\mathcal{M}_{g, 1}$ при всех значениях $g$ тремя способами: с применением улучшенных рекуррентных соотношений Харера-Цагира, с применением разложения гивенталевского типа для матричной модели Концевича-Пеннера и с помощью явного подсчета диаграмм.
\end{abstract}

Ключевые слова: хордовые диаграммы, разложение Гивенталя, матричная модель Концевича-Пеннера, дискретные объемы, пространства модулей, компактификация Делиня-Мамфорда.

DOI: $10.4213 / \operatorname{tmf} 8951$

Разделы 2 и 3, а также п. 6.2 раздела 6 написаны Л. О. Чеховым, разделы 1,4 , 5 и 7, а также оставшаяся часть раздела 6 написаны Й. Э. Андерсеном, П. Норбери и Р. К. Пеннером. Исследование Л. О. Чехова выполнено за счет гранта Российского научного фонда (проект № 14-50-00005) в Математическом институте им. В. А. Стеклова Российской академии наук.

* Center for Quantum Geometry of Moduli Spaces, Århus University, Denmark

${ }^{\dagger}$ California Institute of Technology, Pasadena, USA

${ }^{\ddagger}$ Математический институт им. В. А. Стеклова Российской академии наук, Москва, Россия. E-mail: chekhov@mi.ras.ru

$\S^{\S}$ University of Melbourne, Melbourne, Australia

๑ Institut des Hautes Etudes Scientifiques, Bures-sur-Yvette, France 


\section{1. ВВЕДЕНИЕ}

Многоследовые средние $\left\langle\prod_{i=1}^{s} \operatorname{tr} H^{k_{i}}\right\rangle^{\text {conn }}$ по гауссовым унитарным ансамблям длительное время представляли значительный интерес для исследования. Сначала Харер и Цагир [1] получили линейную рекуррентную формулу для односледового среднего в разложении по родам, что позволило получить ответы для исключительно высоких порядков разложения (недостижимые ни для каких иных методов). Хотя точные представления для $s$-следовых средних в виде $s$-кратных интегралов, справедливые при всех $N$, и были получены Брезаном и Хиками [2] с помощью метода реплик, улучшенного в работе [3], задача построения эффективного разложения по родам на основе этого метода остается открытой. Интерес к многоследовым средним оживился после появления методов топологической рекурсии [4], [5] и квантовых кривых [6]-[8]. Как было показано в нашей работе [9], гауссовы средние связаны посредством так называемой матричной модели Концевича-Пеннера (ММКП) [10], [11] с дискретными объемами открытых пространств модулей и одновременно с производящими функциями инвариантов полей потомков когомологической теории поля [12].

ММКП возникает из явных комбинаторных вычислений. Со времени появления работ [13] и [14] известно, что ММКП эквивалентна эрмитовой матричной модели с потенциалом, времена (константы связи) которого связаны с собственными значениями внешней матрицы ММКП с помощью пребразования типа преобразования Мивы, а размер матриц эрмитовой модели оказывается коэффициентом при логарифмическом члене. В работе [9] утверждается, что ММКП есть интеграл от резольвент гауссовой матричной модели. Резольвенты, содержащие многоследовые гауссовы средние, допускают естественное описание в виде мероморфных (мульти)дифференциалов с нулевыми вычетами над рациональной римановой поверхностью, известной как спектральная кривая, а потому их первообразные суть мероморфные функции на спектральной кривой. Гипотетически эти первообразные связаны (это было доказано в гауссовом случае в работе [7], см. также [15]) с так называемой квантовой кривой, представляющей собой линейное дифференциальное уравнение, являющееся некоммутативным квантованием спектральной кривой. Спектральная и квантовая кривые связаны между собой: волновая функция, возникающая из спектральной кривой, оказывается такой специализацией свободной энергии ММКП, которая удовлетворяет дифференциальному уравнению второго порядка, собственно и представляющему собой квантовую кривую.

Геометрическое содержание ММКП также весьма богато: ее свободная энергия была связана со структурами дискретизованных пространств модулей в работе [16], а недавно она была связана (см. работы [17] и [18]) с производящими функциями дискретных объемов $N_{g, s}\left(P_{1}, \ldots, P_{s}\right)$ - квазимногочленами, введенными в работе [19], которые позволяют вычислить число целых точек во внутренности $\mathcal{M}_{g, s}$ пространств модулей римановых поверхностей рода $g$ с $s>0$ дырками, периметры которых $P_{j} \in \mathbb{Z}_{+}, j=1, \ldots, s$, фиксированы в униформизации Штребеля. Более того, в работе [20] было показано, что в специальных временах $T_{2 n}^{ \pm}$, задаваемых дискретными преобразованиями Лапласа от мономов $P_{I}^{2 k}$, эта модель допускает разложение в произведение двух моделей Концевича, связанных каноническим преобразованием Боголюбова, что было первым примером формул разложения гивента- 
левского типа [21]. Подход работы [20] будет использован для представления членов разложения свободной энергии $\mathcal{F}_{g, s}$ ММКП в конечные суммы по графам, вершины которых суть члены разложения свободной энергии модели Концевича, при этом внутренние ребра отвечают квадратичным членам в операторе канонического преобразования, внешние полуребра (дилатонные листы) отвечают постоянным сдвигам старших времен и, наконец, внешние ребра (обычные листы) несут времена $T_{2 n}^{ \pm}$. Такое представление в виде графов обеспечивает еще одно доказательство квазиполиномиальности чисел $N_{g, s}\left(P_{1}, \ldots, P_{s}\right)$.

Из работ [22] и [23] известно, что члены топологической рекурсии [4], [24]-[26], основанной на специальной спектральной кривой, удовлетворяющей условиям совместности (связывающим инварианты $w_{0,1}$ и $w_{0,2}$ ), оказываются инвариантами полей потомков когомологической теории поля, или, что эквивалентно, фробениусова многообразия.

Фундаментальное семейство фробениусовых многообразий, описанное Дубровиным [27], задается пространствами Гурвица. Для $\mu=\left(\mu_{1}, \ldots, \mu_{n}\right)$ пространство Гурвица $H_{g, \mu}$ состоит из классов гомотопий разветвленных накрытий сферы поверхностью рода $g$ с $n$ отмеченными точками над $\infty$, профилями ветвлений $\left(\mu_{1}, \ldots, \mu_{n}\right)$ и простыми ветвлениями над $\mathbb{P}^{1}-\infty$. Это пространство имеет размерность $|\mu|+n+$ $2 g-2$, где $|\mu|=\mu_{1}+\cdots+\mu_{n}$.

Двумерное многообразие Гурвица-Фробениуса $H_{0,(1,1)}$ задается разветвленным двойным накрытием сферы с двумя точками ветвления, которое остается неразветвленным в точке бесконечности. Его свободная энергия имеет вид

$$
F_{0}\left(t_{0,1}, t_{0,0}\right)=\frac{1}{2} t_{0,0}^{2} t_{0,1}+\frac{1}{2} t_{0,1}^{2} \ln t_{0,1}-\frac{3}{4} t_{0,1}^{2}
$$

с эйлеровым векторным полем

$$
E=t_{0,0} \frac{\partial}{\partial t_{0,0}}+2 t_{0,1} \frac{\partial}{\partial t_{0,1}} .
$$

Заметим, что выражение (1.1) появляется как стандартный член (пертурбативный вклад) в разложении любой матричной модели, если отождествить $t_{0,1}$ с нормированным числом собственных значений, а $t_{0,0}-\mathrm{c}$ первым временем. При этом получается выражение

$$
\begin{gathered}
\ln \int_{i=1}^{t_{0,1} N} d x_{i} \prod_{i<j}\left(x_{i}-x_{j}\right)^{2} \exp \left[-N \sum_{i=1}^{t_{0,1} N}\left(\frac{1}{2} x_{i}^{2}-t_{0,0} x_{i}\right)\right]= \\
=N^{2} F_{0}\left(t_{0,1}, t_{0,0}\right)+\sum_{g=1}^{\infty} N^{2-2 g} F_{g}\left(t_{0,1}\right),
\end{gathered}
$$

в котором ведущий член $1 / N$-разложения свободной энергии приведенной выше гауссовой матричной модели задается в точности выражением (1.1).

В работе [9] дискретные объемы были соотнесены с гауссовыми средними $W_{s}^{(g)}\left(x_{1}, \ldots, x_{s}\right)$, и с помощью описания в терминах когомологической теории поля удалось связать дискретные объемы с инвариантами полей потомков когомологической теории поля. Эти инварианты вычисляются уже в терминах замкнутых пространств модулей $\mathcal{M}_{g, s}$, компактифицированных по Делиню и Мамфорду. 
Работа имеет следующую структуру.

В разделе 2 устанавливается эквивалентность между гауссовыми средними (корреляционными функциями) и членами разложения свободной энергии ММКП.

В разделе 3 описываются результаты работ [16], [19] и [20] для открытых дискретизованных пространств модулей, которые были использованы в работе [9] для установления связи между приведенными выше гауссовыми средними и дискретными объемами в чисто комбинаторном виде. Квантовую кривую при этом можно получить, рассматривая ММКП в случае матриц единичного размера. Далее приводятся описание формул разложения гивенталевского типа для ММКП, полученных в работе [20], и их представление в виде разложения по графам для членов разложения свободной энергии по родам. Из этого представления в виде графов также вытекает свойство квазиполиномиальности дискретных объемов, равно как и связь с когомологической теорией поля.

В разделе 4 члены разложения гауссовых средних отождествляются с инвариантами полей потомков когомологической теории поля с применением результатов работ [28] и [29]. Получаемое при этом разложение имеет канонический гивенталевский вид. Коэффициенты этого разложения, или образы дискретных преобразований Лапласа для квазимногочленов $N_{g, s}\left(P_{1}, \ldots, P_{s}\right)$, суть специальные коэффициенты $\hat{b}_{\vec{k}, \vec{\beta}}^{(g)}$, которые в определенной мере задают “наиболее экономичный” способ представления членов разложения по родам для $s$-петлевых средних $W_{s}^{(g)}\left(x_{1}, \ldots, x_{s}\right)$ и задаются линейными комбинациями от инвариантов полей потомков когомологической теории поля для соседних уровней.

В разделе 5 развивается метод топологической рекурсии для гауссовых средних, выписываются общие рекуррентные соотношения на коэффициенты $\hat{b}_{\vec{k}, \vec{\beta}}^{(g)}$ и доказывается, что в области допустимости все эти коэффициенты оказываются положительными целыми числами.

Раздел 6 посвящен случаю однопетлевого среднего. Первый поправочный коэффициент $b_{g-2}^{(g)}$ находится тремя различными способами: с помощью модифицированных рекуррентных соотношений Харера-Цагира, с помощью графического представления разложения гивенталевского типа из раздела 3 и с помощью прямого подсчета диаграмм.

\section{2. ЭФФЕКТИВНАЯ МАТРИЧНАЯ МОДЕЛЬ ДЛЯ МНОГОПЕТЛЕВЫХ ГАУССОВЫХ СРЕДНИХ}

Рассмотрим сумму связных хордовых диаграмм, основанных на $s$ остовах, или вставках петель, несущих на себе переменные $u_{i}, i=1, \ldots, s$. Для начала сформулируем описание в терминах эффективной матричной модели для всех вкладов рода $g$ в виде специальных форм - связных ленточных графов рода $g$ с $s$ гранями и вершинами произвольных порядков не ниже третьего. Из формулы для эйлеровой характеристики при этом можно заключить, что при фиксированных $g$ и $s$ имеется только конечное число таких ленточных графов, совокупность которых будем обозначать через $\Gamma_{g, s}$. Множество $\Gamma_{g, s}$ перечисляет клетки в каноническом разложении Штребеля-Пеннера пространства модулей $\mathcal{M}_{g, s}$ по идеальным клеткам. В соответствии с утверждением работы [29] множество $\Gamma_{g, s}$ находится в биекции с циклическими хордовыми диаграммами, которые также называются формами 
в терминологии работы [29], - это хордовые диаграммы, являющиеся зародышевыми и не имеющие ограничивающей хорды.

Корреляционные функции, или средние, задаются интегралами

$$
\left\langle\prod_{i=1}^{s}\left(\operatorname{tr} H^{k_{i}}\right)\right\rangle=\int_{H \in \mathcal{H}_{N}}\left(\prod_{i=1}^{s} \operatorname{tr} H^{k_{i}}\right) e^{-(N / 2) \operatorname{tr} H^{2}} D H,
$$

в которых $\mathcal{H}_{N}$ - множество эрмитовых матриц размера $N \times N$. По теореме Вика произвольная корреляционная функция (2.1) представляется в виде суммы по всем возможным (полным) спариваниям между матричными элементами $M_{i j}$, а сами спаривания задаются двухточечными корреляционными функциями $\left\langle H_{i, j} H_{k, l}\right\rangle=\delta_{i l} \delta_{j k} / 4$. Эти спаривания удобно представить в виде ребер - парных линий индексов. Соответствующие индексные линии проходят вдоль граней ленточных графов, содержащих упорядоченные наборы из $s$ вершин с валентностями $k_{i}, i=1, \ldots, s$, и $\sum_{i=1}^{s} k_{i} / 2$ ребер. В каждой вершине фиксируется циклический порядок ребер, инцидентных этой вершине. Кроме того, в каждой вершине отмечается одно - "первое" - ребро. Обозначим через $\widehat{\Gamma}\left(k_{1}, \ldots, k_{s}\right)$ такую совокупность ленточных графов. Тогда сумма в формуле (2.1) принимает вид

$$
\sum_{\gamma \in \widehat{\Gamma}\left(k_{1}, \ldots, k_{s}\right)} N^{b(\gamma)-\sum_{i=1}^{s} k_{i} / 2},
$$

где $b(\gamma)$ - число граничных компонент графа $\gamma$.

Обозначим через $\widehat{\Gamma}\left(k_{1}, \ldots, k_{s}\right)^{c}$ подмножество связных ленточных графов в $\widehat{\Gamma}\left(k_{1}, \ldots, k_{s}\right)$, и пусть $\left\langle\prod_{i=1}^{s}\left(\operatorname{tr} H^{k_{i}}\right)\right\rangle^{\text {conn } о б о з н а ч а е т ~ т у ~ ч а с т ь ~ с у м м ы, ~ к о т о р а я ~ в к л ю-~}$ чает в себя только связные диаграммы. Связные корреляционные функции при этом допускают $1 / N$-разложение:

$$
N^{s-2}\left\langle\prod_{i=1}^{s}\left(\operatorname{tr} H^{k_{i}}\right)\right\rangle^{\mathrm{conn}}=\sum_{g=0}^{\infty} N^{-2 g}\left\langle\prod_{i=1}^{s}\left(\operatorname{tr} H^{k_{i}}\right)\right\rangle_{g}^{\mathrm{conn}},
$$

в котором слагаемое с

$$
\left\langle\prod_{i=1}^{s}\left(\operatorname{tr} H^{k_{i}}\right)\right\rangle_{g}^{\mathrm{conn}}=\left|\widehat{\Gamma}_{g}\left(k_{1}, \ldots, k_{s}\right)^{c}\right|
$$

отвечает множеству $\widehat{\Gamma}_{g}\left(k_{1}, \ldots, k_{s}\right)^{c}$ связных ленточных графов рода $g$ с штрихованными вершинами (штрихи отмечают первые ребра).

Для случая нештрихованных вершин при этом справедлива следующая формула:

$$
\begin{aligned}
(-1)^{s}\left\langle\prod_{i=1}^{s} \operatorname{tr} \ln \left(1-u_{i} H\right)\right\rangle_{g}^{\mathrm{conn}} & =\sum_{\left\{k_{1}, \ldots, k_{s}\right\} \in \mathbb{Z}_{+}^{s}} \prod_{i=1}^{s}\left(\frac{u_{i}^{k_{i}}}{k_{i}}\right)\left\langle\prod_{i=1}^{s}\left(\operatorname{tr} H^{k_{i}}\right)\right\rangle_{g}^{\mathrm{conn}}= \\
& =\sum_{\left\{k_{1}, \ldots, k_{s}\right\} \in \mathbb{Z}_{+}^{s}} \sum_{\gamma \in \Gamma_{g}\left(k_{1}, \ldots, k_{s}\right)^{c}} \frac{1}{|\operatorname{Aut}(\gamma)|} \prod_{i=1}^{s} u_{i}^{k_{i}},
\end{aligned}
$$

где $\Gamma_{g}\left(k_{1}, \ldots, k_{s}\right)^{c}$ - множество связных ленточных графов рода $g$ с $s$ нештрихованными занумерованными вершинами, имеющими валентности $k_{1}, \ldots, k_{s}$, a $\operatorname{Aut}(\gamma)-$ 


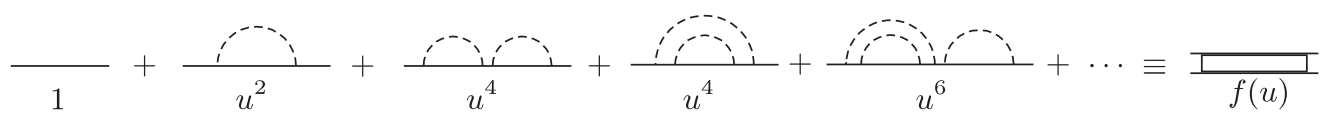

Рис. 1. Суммирование радужных подграфов на хордах (штриховые линии) для одного остова (сплошная линия). В результате получается новое (утолщенное) ребро нового остова.

группа автоморфизмов ленточного графа $\gamma$ с упорядоченными вершинами. Переход от выражений с нештрихованными вершинами к выражениям с штрихованными вершинами, или к хордовым диаграммам, осуществляется посредством дифференцирования:

$$
\begin{aligned}
\left\langle\prod_{i=1}^{s} \operatorname{tr} \frac{1}{I-u_{i} H}\right\rangle_{g}^{\mathrm{conn}} & \equiv\left\langle\prod_{i=1}^{s} \operatorname{tr}\left[\sum_{k_{i}=1}^{\infty} u_{i}^{k_{i}} H^{k_{i}}\right]\right\rangle_{g}^{\mathrm{conn}}= \\
& =(-1)^{s}\left[\prod_{i=1}^{s} u_{i} \frac{\partial}{\partial u_{i}}\right]\left\langle\prod_{i=1}^{s} \operatorname{tr} \ln \left(1-u_{i} H\right)\right\rangle_{g}^{\mathrm{conn}}
\end{aligned}
$$

Формула (2.3) с учетом (2.4) дает

$$
\left[\prod_{i=1}^{s} u_{i} \frac{\partial}{\partial u_{i}}\right]\left\langle\prod_{i=1}^{s} \operatorname{tr} \ln \left(1-u_{i} H\right)\right\rangle_{g}^{\mathrm{conn}}=\sum_{\gamma \in \widehat{\Gamma}_{g, s}^{c}} N^{2-2 g} \prod_{i=1}^{s} u_{i}^{k_{i}} .
$$

\section{1. Суммирование планарных подграфов - переход к эффективной} матричной модели. Произведем сначала частичное пересуммирование по планарным подграфам в выражении (2.3). Планарная хордовая диаграмма на отрезке есть радужная диаграмма (см. примеры на рис. 1). Радужные диаграммы с заданным числом хорд перечисляются числами Каталана, производящая функция которых имеет вид

$$
f\left(u_{i}\right):=\frac{1-\sqrt{1-4 u_{i}^{2}}}{2 u_{i}^{2}},
$$

так что можно в эффективной теории заменить исходное ребро хордовой диаграммы утолщенным ребром, несущим множитель $f\left(u_{i}\right)$, и запретить существование "пузырей", или радужных подграфов.

Перейдем теперь к суммированию подграфов лестничного типа, в которых "перекладина" лестницы соединяет два цикла, несущих (различные или совпадающие) индексы $i$ и $j$ (см. пример на рис. 2). Каждая лестница содержит не менее одной перекладины, представляющей собой хорду, несущую множтель $u_{i} u_{j}$. Эффективный ленточный граф с новыми ребрами и вершинами получается с помощью раздутия циклов утолщенных ребер остовов до тех пор, пока они не соединятся попарно вдоль перекладин (каждое новое ребро содержит не менее одной перекладины); расцепленные части этих циклов составляют при этом циклы длин $2 r_{k} \geqslant 6$, попеременно ограниченных $r_{k}$ перекладинами (хордами) и $r_{k}$ утолщенными ребрами циклических остовов; эти циклы становятся вершинами соответствующих порядков $r_{k} \geqslant 3$ нового ленточного графа. 

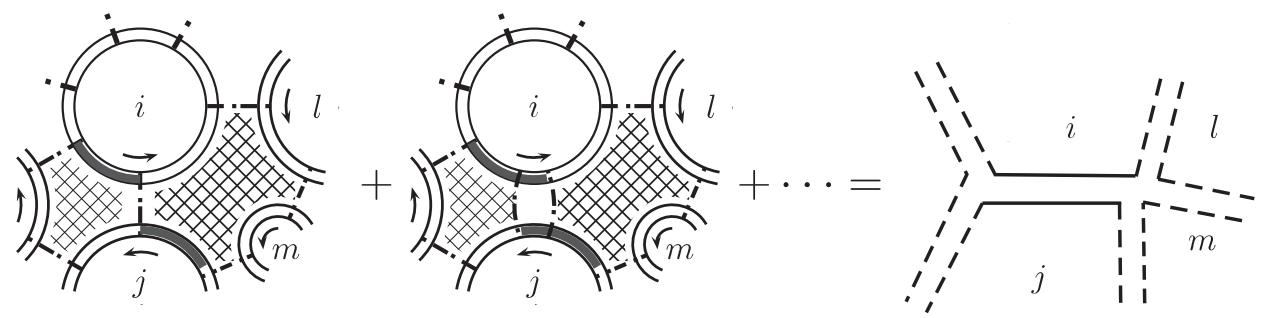

Рис. 2. Пересуммирование для лестничных подграфов. Утолщенные ребра, ассоциированные с выделенной лестничной поддиаграммой, становящейся ребром нового ленточного графа, выделены темным цветом. Крест-накрест штрихованные области становятся соответственно трех- и четырехвалентными вершинами нового ленточного графа, представляющего собой форму.

Вводя переменные $e^{\lambda_{i}}=\frac{1+\sqrt{1-4 u_{i}^{2}}}{2 u_{i}}$ или $u_{i}=\frac{1}{e^{\lambda_{i}}+e^{-\lambda_{i}}}$ для каждого лестничного подграфа, получаем сумму

$$
\sum_{k=1}^{\infty}\left(u_{i} u_{j} f\left(u_{i}\right) f\left(u_{j}\right)\right)^{k}=\frac{1}{\left(u_{i} f\left(u_{i}\right) u_{j} f\left(u_{j}\right)\right)^{-1}-1}:=\frac{1}{e^{\lambda_{i}+\lambda_{j}}-1} .
$$

Таким образом достигается эффективное описание.

ТЕОРема 1 [9]. Член рода g (нештрихованной) связной диаграммы с $s$ остовами задается следующей (конечной!) суммой по ленточным графам форм $\Gamma_{g, s}$ рода $g$ c s гранями и вершинами валентности не менее трех:

$$
\begin{aligned}
\left\langle\prod_{i=1}^{s} \operatorname{tr} \ln \left(e^{\lambda_{i}}+e^{-\lambda_{i}}-H\right)\right\rangle_{g}^{\mathrm{conn}} & =\sum_{\substack{\text { все графы } \\
\gamma \in \Gamma_{g, s}}} \frac{1}{|\operatorname{Aut}(\gamma)|} \prod_{\text {ребра }} \frac{1}{\mathrm{e}^{\lambda_{e}^{(+)}+\lambda_{e}^{(-)}}-1}= \\
& :=F_{s}^{(g)}\left(\lambda_{1}, \ldots, \lambda_{s}\right),
\end{aligned}
$$

где индексы (+) и (-)” обозначают две стороны (грани) ребра е. В правой части величина $F_{s}^{(g)}\left(\lambda_{1}, \ldots, \lambda_{s}\right)$ представляет собой член диаграммного разложения свободной энергии ММКП [10], задаваемой нормированным интегралом по эрмитовым матрицам $X$ размера $N \times N$ :

$$
\begin{aligned}
\mathcal{Z}[\Lambda] & :=\exp \left[\sum_{g, s} N^{2-2 g}\left(\frac{\alpha}{2}\right)^{2-2 g-s} F_{s}^{(g)}(\lambda)\right]= \\
& =\frac{\int D X \exp [-\alpha N \operatorname{tr}(\Lambda X \Lambda X / 4+\ln (1-X) / 2+X / 2)]}{\int D X \exp \left[-\alpha N \operatorname{tr}\left(\Lambda X \Lambda X / 4-X^{2} / 4\right)\right]} .
\end{aligned}
$$

Здесь суммирование ведется по всем стабильным кривым $(2 g+s>2) u \Lambda=$ $\operatorname{diag}\left(e^{\lambda_{1}}, \ldots, e^{\lambda_{N}}\right)$.

Дифференцируя соотношение (2.7) по $\lambda_{i}$, в правой части получим стандартные петлевые средние, или (связные) корреляционные функции $W_{s}^{(g)}\left(x_{1}, \ldots, x_{s}\right), x_{i}=$ 
$e^{\lambda_{i}}+e^{-\lambda_{i}}$, гауссовой матричной модели, которые удовлетворяют стандартным соотношениям топологической рекурсии [4], [24]. Тем самым получаются точные соотношения между резольвентами и членами разложения свободной энергии ММКП:

$$
W_{s}^{(g)}\left(e^{\lambda_{1}}+e^{-\lambda_{1}}, \ldots, e^{\lambda_{s}}+e^{-\lambda_{s}}\right)=\prod_{i=1}^{s}\left[\frac{1}{e^{\lambda_{i}}-e^{-\lambda_{i}}} \frac{\partial}{\partial \lambda_{i}}\right] F_{s}^{(g)}\left(\lambda_{1}, \ldots, \lambda_{s}\right)
$$

Величины $W_{s}^{(g)}\left(x_{1}, \ldots, x_{s}\right)$ здесь подчиняются стандартным соотношениям топологической рекурсии [4], [26] для спектральной кривой $x=e^{\lambda}+e^{-\lambda}, y=\left(e^{\lambda}-e^{-\lambda}\right) / 2$.

\section{3. ММКП И ДИСКРЕТИЗОВАННЫЕ ПРОСТРАНСТВА МОДУЛЕЙ}

3.1. Матричная модель Концевича. Перейдем теперь к описанию клеточного разложения пространств модулей римановых поверхностей рода $g$ с $s>0$ отмеченными точками, доказанного независимо Харером [30] с применением штребелевских дифференциалов [31] и Пеннером [32], [33] с применением гиперболической геометрии. Теорема о клеточном разбиении утверждает, что страты в клеточном разбиении прямого произведения $\mathcal{M}_{g, s} \times \mathbb{R}_{+}^{s}$ открытого пространства модулей и $s$-мерного пространства строго положительных периметров дырок находятся во взаимно однозначном соответствии с ленточными графами рода $g$ с $s$ гранями (эти графы суть формы из раздела 2), ребра которых снабжены строго положительными числами $l_{i} \in \mathbb{R}_{+}$. Периметры $P_{I}, I=1, \ldots, s$, суть суммы чисел $l_{i}$, взятых (с кратностями) по ребрам, составляющим соответствующую грань (граничную компоненту или дырку). Поэтому представляется естественным отождествить эти числа с длинами соответствующих ребер.

Фундаментальная теорема Концевича [34] устанавливает связь между индексами пересечений

$$
\left\langle\tau_{d_{1}} \cdots \tau_{d_{s}}\right\rangle_{g}:=\int_{\overline{\mathcal{M}}_{g, s}} \prod_{I=1}^{s} \psi_{I}^{d_{I}}
$$

и интегралом матричной модели Концевича. Здесь $\psi_{I}-\psi$-класс, или класс Чженя, ассоциированный с $I$-й отмеченной точкой; интегралы от этих классов (индексы пересечений) не зависят от выбранных значений $P_{I}$, представляя собой чисто когомологические объекты. Умножая каждый класс $\psi_{I}^{d_{I}}$ на $P_{I}^{2 d_{I}}$ и производя преобразование Лапласа по всем $P_{I}$, получим

$$
\begin{aligned}
& \iint_{0}^{\infty} d P_{1} \cdots d P_{s} \exp \left[-\sum_{I} P_{I} \lambda_{I}\right] \int_{\mathcal{M}_{g, s}} \prod_{I=1}^{s} P_{I}^{2 d_{I}} \psi_{I}^{d_{I}}= \\
& =\left\langle\tau_{d_{1}} \cdots \tau_{d_{s}}\right\rangle_{g} \prod_{I=1}^{s} \prod_{I=1}^{s} \frac{\left(2 d_{I}\right) !}{\lambda_{I}^{2 d_{I}+1}} .
\end{aligned}
$$

Используя явное представление для $\psi$-классов из работы [34], левую часть выражения (3.1) можно представить в виде суммы по трехвалентным ленточным графам с весами $1 /\left(\lambda_{I_{1}}+\lambda_{I_{2}}\right)$ на ребрах, где $I_{1}$ и $I_{2}-$ индексы двух (возможно, совпадающих) циклов, инцидентных данному ребру. Также появляется множитель $2^{|L|-|V|}$ 
(где через $|V|$ и $|L|$ обозначены числа вершин и ребер графа). Производящая функция при этом задается знаменитым интегралом матричной модели Концевича:

$$
\begin{gathered}
\exp \left[\sum_{g=0}^{\infty} \sum_{s=1}^{\infty} N^{2-2 g} \alpha^{2-2 g-s} \mathcal{F}_{\mathrm{K}}^{(g, s)}\left(\left\{\xi_{k}\right\}\right)\right]:= \\
:=\frac{\int D X \exp \left[-\alpha N \operatorname{tr}\left(X^{2} \Lambda / 2+X^{3} / 6\right)\right]}{\int D X \exp \left[-\alpha N \operatorname{tr}\left(X^{2} \Lambda / 2\right)\right]}
\end{gathered}
$$

где

$$
\xi_{k}:=\frac{1}{N} \sum_{i=1}^{N} \frac{(2 k) !}{\lambda_{i}^{2 k+1}}=\frac{1}{N} \sum_{i=1}^{N} \int_{0}^{\infty} d P_{I} P_{I}^{2 k} e^{-\lambda_{I} P_{I}}
$$

- времена матричной модели Концевича.

3.2. Открытые дискретизованные пространства модулей и ММКП. Как было предложено в работе [16], положим все длины ребер графов Пеннера-Штребеля неотрицательными целыми числами $l_{i} \in \mathbb{Z}_{+}, i=1, \ldots,|L| \leqslant 6 g-6+3 s$. Вместо интегрирований по $\mathcal{M}_{g, s}$ будем производить суммирования по целым точкам во внутренности пространства $\mathcal{M}_{g, s}$.

Так как длина $l_{i}$ каждого ребра входит ровно два раза в сумму $\sum_{I=1}^{s} P_{I}$, эта сумма всегда оказывается положительным четным числом, и это ограничение должно учитываться при выполнении дискретного преобразования Лапласа с мерой $\exp \left[-\sum_{I=1}^{s} \lambda_{I} P_{I}\right]$. По аналогии с непрерывным преобразованием Лапласа в модели Концевича введем новые переменные времен, представляющие собой дискретные преобразования Лапласа:

$$
T_{2 k}^{ \pm}\left(\lambda_{I}\right):=\frac{\partial^{2 k}}{\partial \lambda_{I}^{2 k}} \frac{1}{\mp e^{\lambda_{I}}-1}=\sum_{P_{I}=1}^{\infty}(\mp 1)^{P_{I}} P_{I}^{2 k} e^{-\lambda_{I} P_{I}}
$$

ограничения на $\mathbb{Z}_{2}$ обеспечивают существование двух наборов времен.

Следуя работе [19], мы определили дискретные обгемы $N_{g, s}\left(P_{1}, \ldots, P_{s}\right)$ - взвешенные числа во внутренностях пространств модулей $\mathcal{M}_{g, s}^{\text {disc }} \times \mathbb{Z}_{+}^{s}$ с фиксированными положительными целыми числами $P_{I}, I=1, \ldots, s$, являющимися периметрами дырок (циклов). Эти дискретные объемы равны (по модулю стандартных множителей объемов групп автоморфизмов) числам всех ленточных графов с вершинами валентностей $\geqslant 3$ с положительными целыми длинами ребер, ограниченными условиями, фиксирующими длины всех циклов (периметров). Применяя тождество

$$
\sum_{I=1}^{s} \lambda_{I} P_{I}=\sum_{e \in L} l_{e}\left(\lambda_{I_{1}^{(e)}}+\lambda_{I_{2}^{(e)}}\right)
$$

где $l_{e}$ - длина $e$-го ребра, а $I_{1}^{(e)}$ и $I_{2}^{(e)}-$ индексы двух (возможно совпадающих) циклов, инцидентных $e$-му ребру, получим

$$
\sum_{\left\{P_{I}\right\} \in \mathbb{Z}_{+}^{s}} N_{g, s}\left(P_{1}, \ldots, P_{s}\right) \exp \left[-\sum_{I=1}^{s} P_{I} \lambda_{I}\right]=\sum_{\Gamma_{g, s}} \frac{1}{\left|\operatorname{Aut} \Gamma_{g, s}\right|} \prod_{e=1}^{|L|} \frac{1}{e^{\lambda_{\left.I_{1}^{(}\right)}+\lambda_{I_{2}(e)}}-1} .
$$


В выражении (3.5) нетрудно узнать член разложения по родам ММКП (2.8). Справедлива следующая лемма.

ЛЕмма 1 [9]. Производящая функиия преобразованных по Лапласу дискретных обгемов $N_{g, s}\left(P_{1}, \ldots, P_{s}\right)$ задается ММKП (2.8). Соответствие с (3.5) достигается с помощъю формуль

$$
\begin{gathered}
\exp \left[\sum_{g, s, P_{j} \in \mathbb{Z}_{+}}^{\prime} N^{2-2 g} \alpha^{2-2 g-s} N_{g, s}\left(P_{1}, \ldots, P_{s}\right)\right] \exp \left[-\sum_{I=1}^{s} P_{I} \lambda_{I}\right]= \\
=\frac{\int D X \exp [-\alpha N \operatorname{tr}(\Lambda X \Lambda X / 2+\ln (1-X)+X)]}{\int D X \exp \left[-\alpha N \operatorname{tr}\left(\Lambda X \Lambda / 2 X-X^{2} / 2\right)\right]}
\end{gathered}
$$

в которой суммирование идет по всем стабильным кривым с $2 g-2+s>0$ и со строго положительными периметрами $P_{l}$.

ЗАмЕчаниЕ 1. Формула (3.6) справедлива при всех значениях $N$ и $\lambda_{l}$. Применяя ее в случае $N=1$ (когда матричный интеграл превращается в обычный интеграл) и полагая $\lambda_{l}=\lambda, \alpha=1 / \hbar$ и $x=e^{\lambda}+e^{-\lambda}$, получим

$$
\begin{gathered}
\exp \left[\sum_{g, s, P_{j} \in \mathbb{Z}_{+}}^{\prime} \hbar^{2 g+s-2} N_{g, s}\left(P_{1}^{2}, \ldots, P_{s}^{2}\right) \exp \left[-\lambda \sum_{I=1}^{s} P_{I}\right]\right]= \\
=\sqrt{\frac{1-e^{-2 \lambda}}{\pi \hbar}} e^{-(2 \hbar)^{-1} e^{2 \lambda}+\hbar^{-1} \lambda} F(\hbar, x),
\end{gathered}
$$

где функция

$$
F(\hbar, x):=\int_{-\infty}^{\infty} d t e^{-(1 / \hbar)\left(t^{2} / 2+x t+\ln t\right)}
$$

удовлетворяет дифференциальному уравнению второго порядка

$$
\left[\hbar^{2} \frac{\partial^{2}}{\partial x^{2}}+x \hbar \frac{\partial}{\partial x}+(1-\hbar)\right] F(\hbar, x)=0 .
$$

Тем самым воспроизводится уравнение квантовой кривой из работы [8].

Заметим, что дискретные объемы суть квазимногочлены: их коэффициенты зависят от взаимных четностей периметров $P_{I}$; ниже приводится еще одно доказательство этого факта (см. следствие 1). Поскольку производящая функция (2.8) связана соотношением (2.9) с обычными $s$-петлевыми гауссовыми средними $W_{s}^{(g)}$, имеет место следующая лемма.

Лемма 2 [9]. Корреляиионнъе функиии $W_{s}^{(g)}\left(x_{1}, \ldots, x_{s}\right)$ гауссовой матричной модели, подчиняющейся стандартной топологической рекурсии, основанной на спектральной кривой $x=e^{\lambda}+e^{-\lambda}, y=\left(e^{\lambda}-e^{-\lambda}\right) / 2$, связаны с дискретными обгемами следуюшим явным соотношением:

$$
\begin{aligned}
W_{s}^{(g)} & \left(e^{\lambda_{1}}+e^{-\lambda_{1}}, \ldots, e^{\lambda_{s}}+e^{-\lambda_{s}}\right)= \\
& =\prod_{I=1}^{s}\left[\frac{1}{e^{\lambda_{I}}-e^{-\lambda_{I}}} \sum_{P_{I}=1}^{\infty} P_{I} e^{-P_{I} \lambda_{I}}\right] N_{g, s}\left(P_{1}, \ldots, P_{s}\right) .
\end{aligned}
$$


Матричная модель (2.8) проявляет целый ряд замечательных свойств. Помимо того что она задает производящие функции дискретных объемов, связанных с гауссовыми средними, она также эквивалентна [13], [35] эрмитовой матричной модели с потенциалом, задаваемым преобразованием Мивы переменных $t_{k}=$ $(1 / k) \operatorname{tr}\left(e^{\Lambda}+e^{-\Lambda}\right)^{-k}+\delta_{k, 2} / 2$, она задает производящую функцию числа чистых функций Белого или соответствующих детских рисунков Гротендика [36] (см. также [37]), и, наконец, в специальных временах $T_{2 r}^{ \pm}, r=0,1, \ldots$ (3.4), она эквивалентна произведению двух матричных моделей Концевича [34], переплетенных с помощью канонического преобразования переменных. Обратимся к этому последнему свойству.

ЛЕмма 3 [20]. Статистическая сумма $\mathcal{Z}[\Lambda]$ (2.8), выраженная в терминах переменных времен $T_{k}^{ \pm}(\lambda)(3.4)$, зависит только от четных времен $T_{2 k}^{ \pm}(\lambda)$ и подчиняется следуюшим точным соотношениям:

$$
\mathcal{Z}[\Lambda]=e^{\mathcal{F}_{K P}\left[\left\{T_{2 n}^{ \pm}\right\}\right]}=e^{C(\alpha N)} e^{-N^{-2} \mathcal{A}} e^{\mathcal{F}_{K}\left[\left\{T_{2 n}^{+}\right\}\right]+\mathcal{F}_{K}\left[\left\{T_{2 n}^{-}\right\}\right]}
$$

в которых $\mathcal{F}_{K}\left[\left\{T_{2 n}^{ \pm}\right\}\right]$- свободная энергия матричной модели Концевича (3.2), переменные $T_{2 n}^{ \pm}$, задаваемые формулами (3.4), оказываются тем самым временами иерархий Кортевега-де Фриза, а $\mathcal{A}$ - оператор канонического преобразования:

$$
\begin{aligned}
\mathcal{A}= & \sum_{m, n=0}^{\infty} \frac{B_{2(n+m+1)}}{4(n+m+1)} \frac{1}{(2 n+1) !(2 m+1) !} \times \\
& \times\left\{\frac{\partial^{2}}{\partial T_{2 n}^{+} \partial T_{2 m}^{+}}+\frac{\partial^{2}}{\partial T_{2 n}^{-} \partial T_{2 m}^{-}}+2\left(2^{2(n+m+1)}-1\right) \frac{\partial^{2}}{\partial T_{2 n}^{+} \partial T_{2 m}^{-}}\right\}+ \\
& +\sum_{n=2}^{\infty} \alpha N^{2} \frac{2^{2 n-1}}{(2 n+1) !}\left(\frac{\partial}{\partial T_{2 n}^{-}}+\frac{\partial}{\partial T_{2 n}^{+}}\right) .
\end{aligned}
$$

Здесъ $C(\alpha N)$ - функиия, зависящая только от $\alpha N$ и обеспечивающая обращение в нуль $\mathcal{F}_{K P}\left[\left\{T_{2 n}^{ \pm}\right\}\right]=0$ при $T_{2 n}^{ \pm} \equiv 0$, a $B_{2 k}$ - числа Бернулли, порождаемые соотношением

$$
\frac{t}{e^{t}-1}=\sum_{m=0}^{\infty} \frac{B_{m} t^{m}}{m !} \text {. }
$$

Из этого канонического преобразования немедленно получается представление в виде (обычных) графов для членов $\mathcal{F}_{g, s}\left[\left\{T_{2 n}^{ \pm}\right\}\right]$разложения

$$
\mathcal{F}_{\mathrm{KP}}\left[\left\{T_{2 n}^{ \pm}\right\}\right]=\sum_{g, s} N^{2-2 g} \alpha^{2-2 g-s} \mathcal{F}_{g, s}\left[\left\{T_{2 n}^{ \pm}\right\}\right] .
$$

ЛЕмма 4 [9], [20]. Член $\mathcal{F}_{g, s}\left[\left\{T_{2 n}^{ \pm}\right\}\right]$разложения по родам ММКП (2.8) представляется в виде конечной суммы графов $G_{g, s}$, описанной ниже; каждый граф привносит в сумму множитель, также описанный ниже, деленный на порядок своей группы автоморфизмов:

- каждой вершине $v_{i}, i=1, \ldots, q$, графа $G_{g, s}$ присваиваются маркировка “+” или “-", род $g_{i} \geqslant 0$ и $s_{i}$ концов ребер, входящих в данную вериину $\left(2 g_{i}-2+\right.$ $s_{i}>0$, m.е. все вериины должны отвечать стабильным кривым); каждому кониу ребра присваивается неотрицательное целое число $k_{r, i}^{ \pm}, r=1, \ldots, s_{i}$; на эти целые числа налагается ограничение $\sum_{r=1}^{s_{i}} k_{r, i}^{ \pm}=3 g_{i}-3+s_{i}$, а верхний индекс + или - задается маркировкой вериины; 
- ребра могут бъть или внешними ребрами (объчные листь) с $k_{r, i}^{ \pm} \geqslant 0$ (через $a_{i} \geqslant 0$ будем обозначать число таких внешних ребер, входящих в $i$-ю вериину), или полуребрами (дилатонные листь) с $k_{r, i}^{ \pm} \geqslant 2$ (обозначим через $b_{i} \geqslant 0$ число таких листов, входящих в $i$-ю вершину), или внутренними ребрами, инцидентными двум различным вершинам или одной и той же вершине (их концевые точки несут в общем случае разные числа $k_{r_{1}, i_{1}}^{ \pm} u k_{r_{2}, i_{2}}^{ \pm}$) (обозначим через $l_{i}$ число концевых точек внутренних ребер, иниидентных $i$-й вершине);

- каждая вершина привносит индекс пересечений Концевича $\left\langle\tau_{k_{1, i}^{ \pm}} \cdots \tau_{k_{s_{i}, i}^{ \pm}}\right\rangle g_{i}$;

- каждое внутреннее ребро с маркировкой концов $\left(k_{1}^{+}, k_{2}^{+}\right)$или $\left(k_{1}^{-}, k_{2}^{-}\right)$(концевые точки такого ребра могут быть инцидентными одной и той же вершине) дает множитель

$$
-\frac{B_{2\left(k_{1}^{ \pm}+k_{2}^{ \pm}+1\right)}}{2\left(k_{1}^{ \pm}+k_{2}^{ \pm}+1\right)} \frac{1}{\left(2 k_{1}^{ \pm}+1\right) !\left(2 k_{2}^{ \pm}+1\right) !},
$$

а каждое внутреннее ребро с маркировкой конечных точек $\left(k_{1}^{+}, k_{2}^{-}\right)$(две конечные точки такого ребра обязательно инцидентны двум различным вершинам с различными маркировками $+u-)$ дает множитель

$$
-\frac{B_{2\left(k_{1}^{+}+k_{2}^{-}+1\right)}}{2\left(k_{1}^{+}+k_{2}^{-}+1\right)} \frac{2^{2\left(k_{1}^{+}+k_{2}^{-}+1\right)}-1}{\left(2 k_{1}^{+}+1\right) !\left(2 k_{2}^{-}+1\right) !}
$$

- каждое полуребро с маркировкой $r^{ \pm} \geqslant 2$ дает мноитель $-2^{2 r^{ \pm}}-1 /\left(2 r^{ \pm}+1\right)$ !;

- каждое внешнее ребро с маркировкой $k_{r, i}^{ \pm}$несет на себе соответствующее время $T_{2 k_{r, i}^{ \pm}}^{ \pm}$

- $\sum_{i=1}^{q}\left(g_{i}+l_{i} / 2-1\right)+1=g$ (полный род g равен сумме внутренних родов плюс число петель в графе);

- $\sum_{i=1}^{q} a_{i}=s$ (полное число внешних ребер фиксированно и равно $s$ );

Из приведенных выше формул следует, что

$$
\sum_{j=1}^{s} k_{j}^{\text {ext }}=3 g-3+s-\sum_{j=1}^{|L|}\left(1+k_{j, 1}^{\text {int }}++k_{j, 2}^{\text {int }}\right)-\sum_{j=1}^{|B|}\left(k_{j}^{\text {half }}-1\right),
$$

где без учета маркировок вершин $k_{j}^{\text {ext }} \geqslant 0$ - индексы внешних ребер, $k_{j, 1}^{\text {int }} \geqslant 0 u k_{j, 2}^{\text {int }} \geqslant$ 0 - индексы кониов внутренних ребер, $k_{j}^{\text {half }} \geqslant 2$ - индексы полуребер, $a|L| u|B|-$ числа соответственно внутренних ребер и полуребер графа.

Доказательство этого утверждения состоит в еще одном применении теоремы Вика, теперь с использованием экспоненты от линейно-квадратичного дифференциального оператора (3.9); типичный вид члена такой суммы приведен на рис. 3.

Из леммы непосредственно вытекает

СлеДСТвИЕ 1. Величины $\mathcal{F}_{g, s}\left[\left\{T_{2 n}^{ \pm}\right\}\right]$суть такие многочленъ, что для каждого монома $T_{2 n_{1}}^{+} \cdots T_{2 n_{s}}^{-}$имеем $\sum_{i=1}^{s} n_{i} \leqslant 3 g-3+s$, с старший член $c \sum_{i=1}^{s} n_{i}=3 g-3+s$ ecms

$$
\left\langle\tau_{n_{1}} \cdots \tau_{n_{s}}\right\rangle_{g}\left(\prod_{i=1}^{s} T_{2 n_{i}}^{+}+\prod_{i=1}^{s} T_{2 n_{i}}^{-}\right)
$$




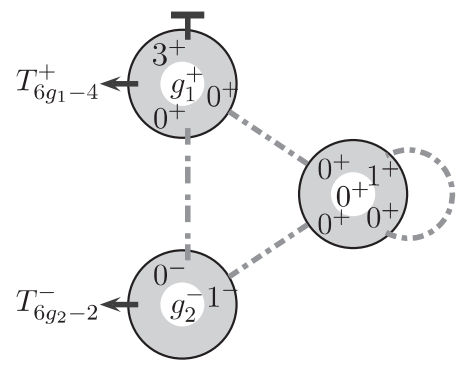

Рис. 3. Типичная диаграмма из разложения по графам $G_{g, s}$.

Отсюда также следует, что все дискретные обгеми $N_{g, s}\left(P_{1}, \ldots, P_{s}\right)$ являются $\mathbb{Z}_{2}$-квазимногочленами относительно переменных $P_{I}^{2}$.

ДоказАтельство. Дискретные объемы $N_{g, s}\left(P_{1}, \ldots, P_{s}\right)$ зависят только от четных степеней $P_{I}$, поскольку $\mathcal{F}_{g, s}$ зависят только от четных времен $T_{2 n}^{ \pm}$; квазиполиномиальность непосредственно следует из того, что $\mathcal{F}_{g, s}-$ многочлены от $T_{2 n}^{+}$и $T_{2 n}^{-}$.

ЗАмечАниЕ 2. Заметим, что квадратичная часть дифференциального оператора (3.9) имеет знакочередующуюся структуру, так как числа Бернулли $B_{2 n}$ положительны при нечетных $n$ и отрицательны при четных $n$,

$$
B_{2 n}=(-1)^{n+1} \frac{2(2 n) !}{(2 \pi)^{2 n}}\left[1+\frac{1}{2^{2 n}}+\frac{1}{3^{2 n}}+\cdots\right] .
$$

3.3. Времена для мультирезольвент. Рассмотрим сначала величины $N_{g, 1}(P)$, которые представляют собой многочлены степени $3 g-2$ от переменной $P^{2}$, отличны от нуля только для четных $P$ и должны обращаться в нуль при всех $P=2, \ldots, 4 g-2$ (так как минимальное число ребер для формы рода $g$ с одной гранью равно $2 g$, а минимальное ненулевое значение периметра $P$ равно $4 g$ ). Поэтому для четных $P$ многочлен $N_{g, 1}(P)$ имеет вид $\prod_{k=1}^{2 g-1}\left(P^{2}-(2 k)^{2}\right) \operatorname{Pol}_{g-1}\left(P^{2}\right)$, где $\operatorname{Pol}_{g-1}(x)$ - многочлен степени $g-1$, и $N_{g, 1}(P)$ обращается в нуль при нечетных $P$, так что его преобразование Лапласа в формуле (3.7) имеет вид

$$
\begin{aligned}
& W_{1}^{(g)}\left(e^{\lambda}+e^{-\lambda}\right)= \\
& \quad=\frac{-1}{e^{\lambda}-e^{-\lambda}} \sum_{i=0}^{g-1} \frac{b_{i}^{(g)}}{2^{4 g+2 i-1}(4 g+2 i-1) !} \prod_{k=1}^{2 g+i-1}\left(\frac{\partial^{2}}{\partial \lambda^{2}}-(2 k)^{2}\right) \frac{\partial}{\partial \lambda} \frac{1}{e^{2 \lambda}-1}
\end{aligned}
$$

с некоторыми коэффициентами $b_{i}^{(g)}$. Применяя соотношение $-\frac{\partial}{\partial \lambda} \frac{1}{e^{2 \lambda}-1}=\frac{2}{\left(e^{\lambda}-e^{-\lambda}\right)^{2}}$ и формулу

$$
\left(\frac{\partial^{2}}{\partial \lambda^{2}}-m^{2}\right) \frac{1}{\left(e^{\lambda}-e^{-\lambda}\right)^{m}}=\frac{4 m(m+1)}{\left(e^{\lambda}-e^{-\lambda}\right)^{m+2}}, \quad m \geqslant 1,
$$

получаем представление общего вида для однопетлевого среднего:

$$
\begin{aligned}
& W_{1}^{(g)}\left(e^{\lambda}+e^{-\lambda}\right)= \\
& \quad=\frac{1}{e^{\lambda}-e^{-\lambda}} \sum_{i=0}^{g-1} b_{i}^{(g)} \frac{1}{\left(e^{\lambda}-e^{-\lambda}\right)^{4 g+2 i}}=\frac{1}{\left(e^{\lambda}-e^{-\lambda}\right)^{4 g+1}} \sum_{i=0}^{g-1} \frac{b_{i}^{(g)}}{\left(e^{\lambda}-e^{-\lambda}\right)^{2 i}} .
\end{aligned}
$$


В работе [9] была найдена формула перехода между $b_{i}^{(g)}$ и коэффициентами $P_{g, i}$ из работы [38]: свойство целочисленности $b_{i}^{(g)}$ предполагает такое же свойство для $P_{g, i}$ и наоборот, но доказательство гипотезы положительности для величин $P_{g, s}$, выдвинутой в работе [38], требует дополнительных исследований.

Перейдем теперь к случаю общих $s$-резольвент. Из формулы (2.9) имеем, что (стабильные) петлевые средние (т. е. те, для которых $2 g+s-2 \geqslant 1$ ) суть многочлены $W_{s}^{(g)}\left(e^{\lambda_{1}}+e^{-\lambda_{1}}, \ldots, e^{\lambda_{s}}+e^{-\lambda_{s}}\right)=F_{g, s}\left(\left\{t_{2 n_{j}+1}^{ \pm}\left(\lambda_{j}\right)\right\}\right)$ относительно времен, получающихся подстановкой

$$
T_{2 d}^{ \pm} \rightarrow t_{2 d+1}^{ \pm}\left(\lambda_{j}\right):=\frac{1}{e^{\lambda_{j}}-e^{-\lambda_{j}}}\left(\frac{\partial}{\partial \lambda_{j}}\right)^{2 d+1} \frac{1}{e^{\lambda_{i}} \pm 1}
$$

Все времена $t_{2 d+1}^{ \pm}(\lambda)$ строго антисимметричны относительно замен переменных $\lambda \rightarrow-\lambda$.

Справедливы соотношения

$$
t_{2 d+1}^{-}(\lambda)+t_{2 d+1}^{+}(\lambda)=\frac{1}{e^{\lambda}-e^{-\lambda}}\left(\frac{\partial}{\partial \lambda}\right)^{2 d+1} \frac{2}{e^{2 \lambda}-1}=\sum_{j=1}^{d+1} q_{j, d} \frac{1}{\left(e^{\lambda}-e^{-\lambda}\right)^{2 j+1}}
$$

и

$$
t_{2 d+1}^{-}(\lambda)-t_{2 d+1}^{+}(\lambda)=\frac{1}{e^{\lambda}-e^{-\lambda}}\left(\frac{\partial}{\partial \lambda}\right)^{2 d+1} \frac{2}{e^{\lambda}-e^{-\lambda}}=\sum_{j=1}^{d+1} \tilde{q}_{j, d} \frac{e^{\lambda}+e^{-\lambda}}{\left(e^{\lambda}-e^{-\lambda}\right)^{2 j+1}}
$$

с некоторыми целочисленными коэффициентами $q_{j, d}$ и $\tilde{q}_{j, d}$, где соотношение (3.16) вытекает из равенства $\frac{1}{e^{\lambda}-1}+\frac{1}{e^{\lambda}+1}=\frac{2}{e^{\lambda}-e^{-\lambda}}$ и из еще одного полезного соотношения

$$
\begin{aligned}
& \frac{1}{e^{\lambda}-e^{-\lambda}} \frac{\partial}{\partial \lambda} \prod_{k=1}^{d}\left(\frac{\partial^{2}}{\partial \lambda^{2}}-(2 k-1)^{2}\right) \frac{2}{e^{\lambda}-e^{-\lambda}}=\frac{1}{e^{\lambda}-e^{-\lambda}} \frac{\partial}{\partial \lambda} \frac{2^{2 d+1}(2 d) !}{\left(e^{\lambda}-e^{-\lambda}\right)^{2 d+1}}= \\
& =-2^{2 d+1}(2 d+1) ! \frac{e^{\lambda}+e^{-\lambda}}{\left(e^{\lambda}-e^{-\lambda}\right)^{2 d+3}} .
\end{aligned}
$$

Теперь с применением (3.11), (3.15) и (3.16) можно эквивалентным образом разложить $F_{g, s}\left(\left\{t_{2 n_{j}+1}^{ \pm}\left(\lambda_{j}\right)\right\}\right)$ по переменным

$$
s_{k, \beta}(\lambda):=\frac{\left(e^{\lambda}+e^{-\lambda}\right)^{\beta}}{\left(e^{\lambda}-e^{-\lambda}\right)^{2 k+3}}, \quad k=0, \ldots, 3 g+s-3, \quad \beta=0,1 .
$$

В разделе 4 показано, что коэффициенты этого разложения представляют собой инварианты полей потомков в когомологической теории поля.

Опишем теперь общую структуру многопетлевых средних.

ЛЕмма 5. Общее выражение для стабильного $(2 g+s-3 \geqslant 0)$ петлевого среднего $W_{s}^{(g)}\left(e^{\lambda_{1}}+e^{-\lambda_{1}}, \ldots, e^{\lambda_{s}}+e^{-\lambda_{s}}\right)$ в терминах переменных $s_{k, \beta}(\lambda)$, определенных формулой (3.18), имеет вид

$$
W_{s}^{(g)}\left(e^{\lambda_{1}}+e^{-\lambda_{1}}, \ldots, e^{\lambda_{s}}+e^{-\lambda_{s}}\right)=\sum_{\vec{k}, \vec{\beta}} \hat{b}_{\vec{k}, \vec{\beta}}^{(g)} \prod_{j=1}^{s} s_{k_{j}, \beta_{j}}\left(\lambda_{j}\right)
$$


где $k_{j}$ и $\beta_{j}$ удовлетворяют условиям

$$
2 g-1+\frac{1}{2} \sum_{j=1}^{s} \beta_{j} \leqslant \sum_{j=1}^{s} k_{j} \leqslant 3 g+s-3, \quad \sum_{j=1}^{s} \beta_{j}=0(\bmod 2) .
$$

Два нестабильных петлевых средних имеют вид

$$
\begin{aligned}
W_{1}^{(0)}\left(e^{\lambda}+e^{-\lambda}\right) & =e^{-\lambda} \\
W_{2}^{(0)}\left(e^{\lambda_{1}}+e^{-\lambda_{1}}, e^{\lambda_{2}}+e^{-\lambda_{2}}\right) & =\prod_{i=1,2} \prod_{j=1,2} \frac{1}{e^{\lambda_{i}}-e^{-\lambda_{j}}} .
\end{aligned}
$$

Докажем ограничения (3.20) с применением двух соображений. Прежде всего, если произвести масштабное преобразование $\lambda_{j} \rightarrow \infty$ однородно для всех $j$, $\lambda_{j} \rightarrow \lambda_{j}+R$, каждое ребро даст множитель $e^{-2 R}$ и еще $s$ множителей добавятся за счет производных. Минимальное число ребер (для формы с одной вершиной) равно $2 g+s-1$, так что минимально возможный множитель равен $e^{(-4 g-3 s+2) R}$, в то время как $s_{k, \beta}(\lambda)$ преобразуются как $e^{(-3-2 k+\beta) R}$, что дает оценку снизу. Верхняя оценка появляется из анализа полюсного поведения при $\lambda_{j}=0$. С одной стороны, $s_{k, \beta}(\lambda) \sim \lambda^{-2 k-3}$ при $\lambda \rightarrow 0$ вне зависимости от параметра $\beta$; с другой стороны, из сравнения с моделью Концевича можно заключить, что полюсная структура производных времен Концевича иерархии Кортевега-де Фриза имеет вид $t_{d_{j}}\left(\lambda_{j}\right) \sim \lambda_{j}^{-2 d_{j}-3}$ с $\sum_{j} d_{j} \leqslant 3 g+s-3$, и потому $\sum_{j} d_{j}=\sum_{j} k_{j}$, что приводит к оценке сверху. То, что сумма $\beta$ множителей должна быть четной, следует из свойства симметричности полного выражения относительно глобальной замены времен $T^{ \pm} \rightarrow T^{\mp}$; при этой замене переменные $s_{k, \beta}(\lambda)$ ведут себя как $s_{k, \beta}(\lambda) \rightarrow(-1)^{\beta} s_{k, \beta}(\lambda)$, так что сумма всех бета-множителей должна быть четной.

В разделе 5 с помощью метода топологической рекурсии показано, что все допустимые соотношением (3.20) коэффициенты $\hat{b}_{\vec{k}, \vec{\beta}}^{(g)}$ оказываются положительными целыми числами (см. теорему 7).

\section{4. КОГОМОЛОГИЧЕСКАЯ ТЕОРИЯ ПОЛЯ, СВЯЗАННАЯ С ДИСКРЕТНЫМИ ОБЪЕМАМИ}

Перейдем теперь к описанию когомологической теории поля, связанной с дискретными объемами. Структура фробениусова многообразия размерности $d$ эквивалентна когомологической теории поля в $d$-мерном векторном пространстве $H$ с базисом $\left\{e_{\alpha}\right\}$ и метрикой $\eta$. Покажем, что квазимногочлены дискретных объемов эквивалентны корреляционным функциям когомологической теории поля, связанным с гурвицевским многообразием Фробениуса $H_{0,(1,1)}$, описанным в разделе 1 . В настоящем разделе приведем два подхода случая рода 0: первый подход имеет конструктивный характер, а второй допускает обобщение на все рода. Из конструктивного подхода также следует, что в данном случае возникает однородная когомологическая теория поля. Примарные корреляционные функции этой когомологической теории поля оказываются виртуальными эйлеровыми характеристиками $\chi\left(\mathcal{M}_{g, n}\right)$ пространств модулей. 
4.1. Когомологические теории поля. Для заданного комплексного векторного пространства $H$, снабженного комплексной метрикой $\eta$, когомологическая теория поля представляет собой последовательность $S_{s}$-эквивариантных линейных отображений

$$
I_{g, s}: H^{\otimes s} \rightarrow H^{*}\left(\overline{\mathcal{M}}_{g, s}\right),
$$

удовлетворяющих следующим условиям совместности относительно включения страт. Произвольное разбиение на два непересекающихся подмножества $I \sqcup J=$ $\{1, \ldots, s\}$ задает отображение $\phi_{I}: \overline{\mathcal{M}}_{g_{1},|I|+1} \times \overline{\mathcal{M}}_{g_{2},|J|+1} \rightarrow \overline{\mathcal{M}}_{g, s}$ такое, что

$$
\phi_{I}^{*} I_{g, s}\left(v_{1} \otimes \cdots \otimes v_{s}\right)=I_{g_{1},|I|+1} \otimes I_{g_{2},|J|+1}\left(\bigotimes_{i \in I} v_{i} \otimes \Delta \otimes \bigotimes_{j \in J} v_{j}\right),
$$

где $\Delta=\sum_{\alpha, \beta} \eta^{\alpha \beta} e_{\alpha} \otimes e_{\beta}$ относительно базиса $\left\{e_{\alpha}\right\}$ пространства $H$. Отображение $\psi: \overline{\mathcal{M}}_{g-1, s+2} \rightarrow \overline{\mathcal{M}}_{g, s}$ индуцирует отображение

$$
\psi^{*} I_{g, s}\left(v_{1} \otimes \cdots \otimes v_{s}\right)=I_{g-1, s+2}\left(v_{1} \otimes \cdots \otimes v_{s} \otimes \Delta\right) .
$$

Трехточечная корреляционная функция $I_{0,3}$ вместе с метрикой $\eta$ индуцирует на пространстве $H$ операцию произведения •

$$
u \bullet v=\sum_{\alpha, \beta} I_{0,3}\left(u \otimes v \otimes e_{\alpha}\right) \eta^{\alpha \beta} e_{\beta},
$$

где $I_{0,3}$ принимает значения в $\mathbb{C}$. Вектор $e_{0}$, удовлетворяющий соотношению

$$
I_{0,3}\left(v_{1} \otimes v_{2} \otimes e_{0}\right)=\eta\left(v_{1} \otimes v_{2}\right) \quad \forall v_{1}, v_{2} \in H,
$$

есть единичный элемент для произведения на $H$.

Дополнительное условие, которому удовлетворяют как рассматриваемая когомологическая теория поля, так и инварианты Громова-Виттена, относится к забывающему отображению при $s \geqslant 3 \pi: \overline{\mathcal{M}}_{g, s+1} \rightarrow \overline{\mathcal{M}}_{g, s}$, которое индуцирует отображение

$$
I_{g, s+1}\left(v_{1} \otimes \cdots \otimes v_{s} \otimes e_{0}\right)=\pi^{*} I_{g, s}\left(v_{1} \otimes \cdots \otimes v_{s}\right) .
$$

4.2. Квазимногочлены и инварианты полей потомков. Дискретные объемы $N_{g, s}\left(P_{1}, \ldots, P_{s}\right)$ суть четные $(\bmod 2)$ квазимногочлены, т. е. они представляют собой четные многочлены на каждом фактор-множестве $2 \mathbb{Z}^{s} \subset \mathbb{Z}^{s}$. Определим базис четных $(\bmod 2)$ квазимногочленов, индуцированный (с помощью тензорного произведения) со следующего базиса функций одной переменной $p_{k, \alpha}(b)$ для $k=0,1,2, \ldots$ и $\alpha=0,1$ :

$$
\begin{gathered}
p_{0,0}(b)=\left\{\begin{array}{ll}
1, & b \text { четно, } \\
0, & b \text { нечетно, }
\end{array} \quad p_{0,1}(b)= \begin{cases}0, & b \text { четно, } \\
1, & b \text { нечетно, }\end{cases} \right. \\
p_{k+1, \alpha}(b)=\sum_{m=0}^{b} m p_{k, \alpha}(m), \quad k \geqslant 0 .
\end{gathered}
$$

Тогда получаем

$$
p_{k, \alpha}(b)=\frac{p_{0, k+\alpha}(b)}{4^{k} k !} \prod_{\substack{k \leqslant m \leqslant k \\ m=k+\alpha(\bmod 2)}}\left(b^{2}-m^{2}\right)
$$


где во втором нижнем индексе имеется в виду $k+\alpha(\bmod 2)$.

Положим $\vec{k}=\left(k_{1}, \ldots, k_{s}\right)$ и $\vec{\alpha}=\left(\alpha_{1}, \ldots, \alpha_{s}\right)$.

Теорема 2. Имеет место соотношение

$$
N_{g, s}\left(P_{1}, \ldots, P_{s}\right)=\sum_{\vec{k}, \vec{\alpha}} c_{\vec{k}, \vec{\alpha}}^{g} \prod_{i=1}^{s} p_{k_{i}, \alpha_{i}}\left(P_{i}\right),
$$

коэффициенты в котором суть инварианты полей потомков:

$$
c_{\vec{k}, \vec{\alpha}}^{g}=\int_{\overline{\mathcal{M}}_{g, s}} I_{g, s}\left(e_{\alpha_{1}^{\prime}} \otimes \cdots \otimes e_{\alpha_{s}^{\prime}}\right) \prod_{i=1}^{s} \psi_{i}^{k_{i}} .
$$

ДокАЗАТЕЛЬСтво состоит в применении результатов работы [23], в которой теории со спектральными кривыми, удовлетворяющими некоторым специальным условиям, были отождествлены с полупростыми когомологическими теориями поля. Результат применения метода работы [23], к сожалению, не имеет конструктивного характера, так что здесь случай рода 0 рассматривается в другом подходе, задающем явную реализацию когомологической теории поля.

4.3. Однородная когомологическая теория поля в случае рода 0 . Примарные корреляционные функции когомологической теории поля имеют вид

$$
Y_{g, s}:=\int_{\overline{\mathcal{M}}_{g, s}} I_{g, s}: H^{\otimes s} \rightarrow \mathbb{C},
$$

и их производящую функцию можно записать в виде

$$
F\left(t_{0}, \ldots, t_{D-1}\right)=\sum N^{2-2 g} \frac{1}{s !} Y_{g, s}=\sum N^{2-2 g} F_{g}
$$

где $\left(t_{0}, \ldots, t_{D-1}\right)$ в пространстве $H^{*}$ - базис, дуальный базису $\left\{e_{0}, \ldots, e_{D-1}\right\}$. Член рода $0\left(F_{0}\right)$ оказывается препотенииалом соответствующей когомологической теории поля.

Теорема 3 (Манин [12], теорема III.4.3). Можно однозначно реконструировать когомологическую теорию поля в случае рода 0 исходя из абстрактных корреляиионных функций.

Компактификация Делиня-Мамфорда $\overline{\mathcal{M}}_{g, s}$ допускает естественную стратификацию, перечисляемую дуальными графами. Дуальный граф $\Sigma \in \overline{\mathcal{M}}_{g, s}$ имеет вершины, отвечающие неприводимым компонентам $\Sigma$ с указанными родами, ребра отвечают особенностям $\Sigma$-типа каспов, а хвосты - ребра с открытыми концами (без вершин на концах) - отвечают каждой отмеченной точке $\Sigma$. Если $\Gamma$ - дуальный граф типа $(g, s)$, то множество кривых $D_{\Gamma}$ с одним и тем же ассоциированным дуальным графом $\Gamma$ образует страт пространства модулей $\overline{\mathcal{M}}_{g, s}$. Замыкание $\bar{D}_{\Gamma}=\cup_{\Gamma^{\prime}<\Gamma} D_{\Gamma^{\prime}}$, в котором частичный порядок задается последовательностью сжимаемых ребер, определяет элемент $H^{*}\left(\overline{\mathcal{M}}_{g, s}\right)$. Киль доказал [39], что $H^{*}\left(\overline{\mathcal{M}}_{0, s}\right)$ порождается $\bar{D}_{\Gamma}$, и предъявил все необходимые соотношения. 
Доказательство теоремы 3 использует тот факт, что

$$
\int_{\bar{D}_{\Gamma}} I_{0, s}\left(v_{1} \otimes \cdots \otimes v_{s}\right)=\bigotimes_{v \in V_{\Gamma}} Y_{0,|v|}\left(\bigotimes_{i=1}^{s} v_{i} \otimes \Delta^{\otimes\left|E_{\Gamma}\right|}\right),
$$

это определяет вычисление когомологического класса на граничных стратах тавтологически из определения когомологической теории поля. Поскольку $H^{*}\left(\overline{\mathcal{M}}_{0, s}\right)$ порождается своими граничными стратами, а соотношения на множестве $H^{*}\left(\overline{\mathcal{M}}_{0, s}\right)$ находятся в соответствии с соотношениями, которым удовлетворяют абстрактные корреляционные функции, этого оказывается достаточно для доказательства теоремы.

В частности, имеются примарные инварианты

$$
\begin{gathered}
Y_{0,3}\left(e_{0} \otimes e_{0} \otimes e_{1}\right)=1=Y_{0,3}\left(e_{1} \otimes e_{1} \otimes e_{1}\right), \\
Y_{0, s}\left(e_{0} \otimes \text { любая комбинация }\right)=0, \quad s>3, \\
Y_{0, s}\left(e_{1}^{\otimes s}\right)=N_{0, s}(0, \ldots, 0)=\chi\left(\mathcal{M}_{0, s}\right), \quad s>3,
\end{gathered}
$$

которые задают когомологическую теорию поля в случае рода 0.

Когомологическая теория поля называется конформной, если ее препотенциал оказывается квазиоднородным относительно эйлеровского векторного поля:

$$
E \cdot F_{0}=(3-d) F_{0}+Q(t),
$$

где $Q$ - квадратный многочлен относительно времен $t=\left(t_{0}, \ldots, t_{D-1}\right)$. Применяя реконструкцию в случае рода 0 в теореме 3 , Манин [12] доказал, что конформная когомологическая теория поля индуцирует следующее условие поднятия для когомологической теории поля в случае рода 0.

Пусть $\xi$ - произвольное векторное поле на пространстве $H$, рассматриваемом как многообразие с координатами $t_{0}, \ldots, t_{D-1} \in H^{*}$. Производная Ли относительно $\xi$ от корреляционных функций когомологической теории поля $I_{g, s}$ индуцирует естественное действие

$$
\begin{aligned}
(\xi \cdot I)_{g, s}\left(v_{1} \otimes \cdots \otimes v_{s}\right)= & \operatorname{deg} I_{g, s}\left(v_{1} \otimes \cdots \otimes v_{s}\right)-\sum_{j=1}^{s} I_{g, s}\left(v_{1} \otimes \cdots \otimes\left[\xi, v_{j}\right] \otimes \cdots \otimes v_{s}\right)+ \\
& +\pi_{*} I_{g, s+1}\left(v_{1} \otimes \cdots \otimes v_{s} \otimes \xi\right),
\end{aligned}
$$

где $\pi: \overline{\mathcal{M}}_{g, s+1} \rightarrow \overline{\mathcal{M}}_{g, s}$ - забывающее отображение, $I_{g, s}-\left(H^{*}\left(\overline{\mathcal{M}}_{0, s}\right)\right.$-значные $)$ тензоры на $H$, а векторное поле $\xi$ действует инфинитезимально на $I_{g, s}$.

Назовем когомологическую теорию поля однородной веса $d$, если

$$
(E \cdot I)_{g, s}=((g-1) d+s) I_{g, s} .
$$

Если препотенциал удовлетворяет условию однородности (4.5), то из доказательства теоремы 3 следует свойство однородности для соответствующей когомологической теории поля в случае рода 0 . Производную Ли бивектора $\Delta$, дуального метрике $\eta$ на $H$, можно вычислить в плоских координатах:

$$
\mathcal{L}_{E} \cdot \Delta=\mathcal{L}_{E} \cdot \eta^{i j} e_{i} \otimes e_{j}=\eta^{i j}\left(\left[E, e_{i}\right] \otimes e_{j}+e_{i} \otimes\left[E, e_{j}\right]\right)=(d-2) \eta^{i j} e_{i} \otimes e_{j}=(d-2) \Delta,
$$

где в качестве плоских координат выбраны координаты из работы [40], относительно которых $\eta=\delta_{i, D-1-i}$ и $E=\sum_{i}\left(\alpha_{i} t_{i}+\beta_{i}\right)\left(\partial / \partial t_{i}\right)$, где $\alpha_{i}+\alpha_{D-1-i}=2-d$. 
4.4. Доказательство теоремы 2 в случае рода 0. Докажем теперь теорему 2 в случае рода 0 . Для этого сконструируем препотенциал исходя из примарных (постоянных) членов в многочленах $N_{0, s}\left(P_{1}, \ldots, P_{s}\right)$, которые однозначно (и конструктивно) определяют когомологическую теорию поля в случае рода 0. Более того, из квазиоднородности препотенциала следует свойство однородности когомологической теории поля. Старшие коэффициенты многочленов $N_{0, s}\left(P_{1}, \ldots, P_{s}\right)$ удовлетворяют условию однородности, из которого следует, что эти коэффициенты суть корреляционные функции однородной когомологической теории поля.

Препотенциал

$$
\begin{aligned}
F_{0}= & \sum \frac{1}{s !} Y_{0, s}=\frac{1}{2} t_{0}^{2} t_{1}+\sum_{s \geqslant 3} \frac{1}{s !} N_{0, s}(\overrightarrow{0}) t_{1}^{s}= \\
& =\frac{1}{2} t_{0}^{2} t_{1}+\frac{1}{2}\left(1+t_{1}\right)^{2} \ln \left(1+t_{1}\right)-\frac{1}{2} t_{1}-\frac{3}{4} t_{1}^{2},
\end{aligned}
$$

собранный из $N_{0, s}(\overrightarrow{0})=(-1)^{s-3}(s-3)$ !, квазиоднороден относительно эйлерова векторного поля $E=t_{0}\left(\partial / \partial t_{0}\right)+2\left(1+t_{1}\right)\left(\partial / \partial t_{1}\right)$ :

$$
E \cdot F_{0}=4 F_{0}+t_{1}^{2}+t_{0}^{2}
$$

Это свойство гарантирует, что когомологическая теория поля в случае рода $0 I_{0, s}$, задаваемая теоремой 3, удовлетворяет условию

$$
\pi_{*} I_{g, s+1}\left(e_{S} \otimes e_{1}\right)=\frac{1}{2}\left(1-g+s-\operatorname{deg}-\sum \alpha_{i_{k}}\right) I_{g, s}\left(e_{S}\right)
$$

где $e_{S}=e_{i_{1}} \otimes \cdots \otimes e_{i_{s}}$, а $\alpha_{0}=1, \alpha_{1}=2$ - коэффициенты поля $E$. Эта когомологическая теория поля также удовлетворяет условию поднятия (4.1).

ТЕОРема 4 [41]. Полупростая однородная когомологическая теория поля с плоской единицей однозначно и явно реконструируется на основе своих данных в случае рода 0 .

Таким образом, если известны примарные инварианты в случае рода $0 N_{0, s}(\overrightarrow{0})$, то существует единственная однородная когомологическая теория поля с плоской единицей, отвечающая этим инвариантам. Ниже показано, что ее корреляционные функции согласуются с коэффициентами $N_{g, s}\left(P_{1}, \ldots, P_{s}\right)$.

Отображение поднятия (4.8) в терминах корреляционных функций имеет следующий вид [9]:

$$
\begin{aligned}
\int_{\overline{\mathcal{M}}_{g, s+1}} I_{g, s+1}\left(e_{S} \otimes e_{1}\right) \prod_{i=1}^{s} \psi_{i}^{k_{i}}= & \left(\sum_{i=1}^{s} \frac{k_{i}}{2}+\chi_{g, s}\right) \int_{\overline{\mathcal{M}}_{g, s}} I_{g, s}\left(e_{S}\right) \prod_{i=1}^{s} \psi_{i}^{k_{i}}+ \\
& +\sum_{j=1}^{s} \int_{\overline{\mathcal{M}}_{g, s}} I_{g, s}\left(e_{S \backslash\{j\}} \otimes e_{j}^{*}\right) \prod_{i=1}^{s} \psi_{i}^{k_{i}-\delta_{i j}} .
\end{aligned}
$$


Условие $E \cdot F_{0}=4 F_{0}+t_{1}^{2}+t_{0}^{2}$ на $N_{0, s}(\overrightarrow{0})$ представляет собой специализацию на случай $g=0$ и $P_{i}=0$ уравнения дивизора [17]:

$$
\begin{aligned}
N_{g, s+1}\left(0, P_{1}, \ldots, P_{s}\right)= & \left.\sum_{j=1}^{s} \sum_{k=1}^{P_{j}-1} k N_{g, s}\left(P_{1}, \ldots, P_{s}\right)\right|_{P_{j}=k}+ \\
& +\left(\frac{1}{2} \sum_{j=1}^{s} P_{j}+\chi_{g, s}\right) N_{g, s}\left(P_{1}, \ldots, P_{s}\right) .
\end{aligned}
$$

Условие поднятия для плоской единицы известно как струнное уравнение на корреляционные функции для $2 g-2+s>0$ :

$$
\int_{\overline{\mathcal{M}}_{g, s+1}} I_{g, s+1}\left(v_{1} \otimes \cdots \otimes v_{s} \otimes e_{0}\right) \prod_{i=1}^{s} \psi_{i}^{k_{i}}=\sum_{j=1}^{s} \int_{\overline{\mathcal{M}}_{g, s}} I_{g, s}\left(v_{1} \otimes \cdots \otimes v_{s}\right) \prod_{i=1}^{s} \psi_{i}^{k_{i}-\delta_{i, j}} ;
$$

это уравнение согласуется с рекуррентным соотношением [17]

$$
N_{g, s+1}\left(1, P_{1}, \ldots, P_{s}\right)=\left.\sum_{j=1}^{s} \sum_{k=1}^{P_{j}} k N_{g, s}\left(P_{1}, \ldots, P_{s}\right)\right|_{P_{j}=k} .
$$

В частности, это соотношение позволяет доказать теорему 2 в случае рода 0, поскольку рекуррентные соотношения (4.9) и (4.10) однозначно определяют корреляционные функции $I_{0, s}$ и $N_{0, s}\left(P_{1}, \ldots, P_{s}\right)$.

Это конструктивное доказательство явно описывает классы $I_{0, s}\left(e_{S}\right) \in H^{*}\left(\overline{\mathcal{M}}_{0, s}\right)$ рода 0:

$$
\int_{\overline{\mathcal{M}}_{0, s}} I_{0, s}\left(e_{S}\right)= \begin{cases}\chi\left(\mathcal{M}_{0, s}\right), & e_{S}=e_{1}^{\otimes s} \\ 0 & \text { в других случаях. }\end{cases}
$$

4.5. Общее доказательство теоремы 2 с применением метода ДунинаБарковского-Орантана-Шадрина-Шпица. Установим теперь соответствие между корреляционными функциями когомологической теории поля и дискретными объемами в старших родах на основе результатов работы [23], в которой показано, что в случае спектральных кривых, удовлетворяющих специальному условию совместности, реконструкцию Гивенталя корреляционных функций старших родов можно сформулировать в терминах графов, и те же самые графы можно применить при вычислении топологической рекурсии.

В работе [23] был применен метод Эйнара из работы [22], с помощью которого произвольной полупростой когомологической теории поля можно поставить в соответствие локальную спектральную кривую $(\Sigma, B, x, y)$. При этом $R$-матрица Гивенталя порождает бидифференциал $B$ на спектральной кривой:

$$
\sum_{p, q} \check{B}_{p, q}^{i, j} z^{p} w^{q}=\frac{1}{z+w}\left[\delta^{i j}-\sum_{k=1}^{N} R_{k}^{i}(-z) R_{k}^{j}(-w)\right]
$$

где $\check{B}_{p, q}^{i, j}$ - коэффициенты асимптотического разложения преобразования Лапласа регулярной части бидифференциала Бергмана $B$, выраженного в терминах локальных координат $s_{i}=\sqrt{x-x\left(a_{i}\right)}$ при $d x\left(a_{i}\right)=0$. С помощью $R$-матрицы и матрицы 
перехода $\Psi$ из базиса плоских координат в нормированный канонический базис мероморфный дифференциал $y d x$ можно выразить в терминах $s_{i}$. В частности, отсюда следует условие совместности (4.13) между дифференциалом $y d x$ и бидифференциалом $B$.

Метод работы [23] можно применять в любом из двух направлений, начиная или с полупростой когомологической теории поля, или со спектральной кривой. Препотенциал $F_{0}(4.7)$ задает полупростую когомологическую теорию поля, тем самым порождая $R$-матрицу и матрицу перехода $\Psi$, а потому и собственно спектральную кривую. Однако, имея в наличии кандидата на роль спектральной кривой, можно начать со спектральной кривой и применить метод работы [23] для получения коэффициентов $N_{g, s}\left(P_{1}, \ldots, P_{s}\right)$ как инвариантов полей потомков когомологической теории поля. Поскольку эта процедура согласуется с приведенной выше когомологической теорией поля в случае рода 0 , в силу однозначности реконструкции такая процедура приводит в итоге к той же самой когомологической теории поля, что и теория, следующая из теоремы Телемана.

Спектральные кривые для дискретных объемов и для инвариантов ГромоваВиттена для $\mathbb{P}^{1}$ имеют похожие структуры:

$$
\begin{array}{ll}
\text { дискретные объемы } & x=z+\frac{1}{z}, \quad y=z, \quad B=\frac{d z d z^{\prime}}{\left(z-z^{\prime}\right)^{2},} \\
\text { инварианты Громова-Виттена } & x=z+\frac{1}{z}, \quad y=\ln z, \quad B=\frac{d z d z^{\prime}}{\left(z-z^{\prime}\right)^{2}},
\end{array}
$$

и так как величины $x$ и $B$ задают $R$-матрицу однозначно, она совпадает для обеих кривых. $R$-матрица для инвариантов Громова-Виттена на проективной кривой $\mathbb{P}^{1}$ имеет вид [23]

$$
R(u)=\sum_{k=0}^{\infty} R_{k} u^{k}, \quad R_{k}=\frac{(2 k-1) ! !(2 k-3) ! !}{2^{4 k} k !}\left(\begin{array}{cc}
-1 & (-1)^{k+1} 2 k i \\
2 k i & (-1)^{k+1}
\end{array}\right) .
$$

Результаты работы [23] применимы к тем спектральным кривым, для которых преобразование Лапласа дифференциала $y d x$ связано с этой $R$-матрицей (являющейся по сути преобразованием Лапласа от регулярной части бидифференциала).

В локальных координатах $s_{i}, i=1,2$, вблизи $x= \pm 2$, задаваемых выражениями $x=s_{i}^{2} \pm 2$, имеем

$$
\begin{aligned}
& y=1+s_{1}+\frac{1}{2} s_{1}^{2}+\sum_{k=1}^{\infty}(-1)^{k-1} \frac{(2 k-3) ! !}{2^{3 k} k !} s_{1}^{2 k+1} \\
& y=-1+i s_{2}+\frac{1}{2} s_{2}^{2}-i \sum_{k=1}^{\infty} \frac{(2 k-3) ! !}{2^{3 k} k !} s_{2}^{2 k+1}
\end{aligned}
$$

так что получаем

$$
\begin{aligned}
& (y \check{d} x)_{1}=\frac{\sqrt{u}}{2 \sqrt{\pi}} \int_{\gamma_{1}} e^{-u(x-2)} y d x \sim \sum_{k=0}^{\infty}(-1)^{k-1} \frac{(2 k+1) ! !(2 k-3) ! !}{2^{4 k+1} k !} u^{-(k+1)}, \\
& (y \check{d} x)_{2}=\frac{\sqrt{u}}{2 \sqrt{\pi}} \int_{\gamma_{2}} e^{-u(x+2)} y d x \sim-i \sum_{k=0}^{\infty} \frac{(2 k+1) ! !(2 k-3) ! !}{2^{4 k+1} k !} u^{-(k+1)},
\end{aligned}
$$


где $(-1) ! !=1,(-3) ! !=-1$, а символом $\sim$ обозначено асимптотическое соответствие Пуанкаре по отношению к параметру $u$.

Условие совместности дифференциала $y d x$ и бидифференциала $B$ имеет вид

$$
\frac{1}{\sqrt{2}}\left(\begin{array}{ll}
1 & i
\end{array}\right) \cdot \frac{1}{\sqrt{2}} R(u)=\left(\begin{array}{ll}
(y \check{d} x)_{1} \quad(y \check{d} x)_{2}
\end{array}\right)
$$

где используется первый ряд матрицы перехода $\Psi=\frac{1}{\sqrt{2}}\left(\begin{array}{cc}1 & i \\ 1 & -i\end{array}\right)$. Прямой подсчет показывает, что это условие выполняется при $x=z+1 / z, y=z, B=d z d z^{\prime} /\left(z-z^{\prime}\right)^{2}$.

В этом случае вычисления работы [23] дают набор времен

$$
\xi_{0}^{0}=\frac{1}{2}\left(\frac{1}{1-z}-\frac{1}{1+z}\right), \quad \xi_{0}^{1}=\frac{1}{2}\left(\frac{1}{1-z}+\frac{1}{1+z}\right), \quad \xi_{k}^{i}=\left(\frac{d}{d x}\right)^{k} \xi_{0}^{i}=\sum_{k} p_{k, i} z^{k}
$$

и приводят к основному результату:

$$
W_{s}^{(g)}\left(x_{1}, \ldots, x_{s}\right)=\sum_{\vec{k}, \vec{\alpha}} c_{\vec{k}, \vec{\alpha}}^{g} \prod_{i=1}^{n} \xi_{k_{i}, \alpha_{i}}
$$

где коэффициенты суть инварианты полей потомков (4.3). Как было отмечено выше, когомологическая теория поля, получаемая таким образом, с необходимостью совпадает с однородной когомологической теорией поля, построенной на основе теоремы Телемана, поскольку обе эти теории используют реконструкцию Гивенталя и имеют одни и те же начальные данные.

4.6. Инварианты полей потомков и гауссовы средние. Лемма 2 и формулы (3.11) и (3.17) непосредственно выражают петлевые средние в терминах инвариантов полей потомков.

Теорема 5. Имеет место следующее явное соотношение между инвариантами полей потомков (4.3) когомологической теории поля и гауссовыми средними:

$$
W_{s}^{(g)}\left(e^{\lambda_{1}}+e^{-\lambda_{1}}, \ldots, e^{\lambda_{s}}+e^{-\lambda_{s}}\right)=\sum_{\vec{k}, \vec{\alpha}} c_{\vec{k}, \vec{\alpha}}^{g} \prod_{j=1}^{s} \widehat{p}_{k_{j}, \alpha_{j}}\left(\lambda_{j}\right)
$$

¿əe

$$
\widehat{p}_{k, \alpha}(\lambda)= \begin{cases}2^{1-2 r}(2 r+1) s_{r, 0}(\lambda), & k=2 r, \alpha=0 \\ 2^{-2 r}(2 r+1) s_{r, 1}(\lambda), & k=2 r, \alpha=1 \\ 2^{-2 r+2} 2 r(2 r+1) s_{r, 1}(\lambda), & k=2 r-1, \alpha=0 \\ 2^{-2 r-1} s_{r, 0}(\lambda), & k=2 r+1, \alpha=1\end{cases}
$$

u $s_{r, \beta}(\lambda), \beta=0,1$, заданы соотношениями (3.18).

Пример 1. Топологическая часть (нулевого уровня) когомологической теории поля имеет вид

$$
I_{g, s}\left(e_{\alpha_{1}} \otimes \cdots \otimes e_{\alpha_{s}}\right)=\epsilon(\vec{\alpha}) 2^{g}+\text { члены старших порядков, }
$$

где $\epsilon(\vec{\alpha}) \equiv \sum_{i=1}^{s} \alpha_{i}(\bmod 2)$ равно нулю или единице. Это проясняет асимптотическое поведение топологических инвариантов $W_{s}^{(g)}$ в их полюсах. 
Пример 2. Если $\left\{e_{0}, e_{1}\right\}$ - базис пространства $H$, отвечающий плоским координатам, то

$$
\int_{\overline{\mathcal{M}}_{g, s}} I_{g, s}\left(e_{1}^{\otimes s}\right)=\chi\left(\mathcal{M}_{g, s}\right) .
$$

Это утверждение следует из того, что $N_{g, s}(0,0, \ldots, 0)=\chi\left(\mathcal{M}_{g, s}\right)$ и что

$$
p_{k, \alpha}(0)= \begin{cases}1, & (k, \alpha)=(0,0) \\ 0 & \text { в других случаях. }\end{cases}
$$

Таким образом, можно отождествить коэффициенты $\hat{b}_{\vec{k}, \vec{\beta}}^{g}$ разложений (3.19) с линейными комбинациями инвариантов полей потомков $c_{\vec{k}, \vec{\alpha}}^{g}$ с применением отождествления (4.16): при $s=1$ имеем

$$
\begin{aligned}
& \hat{b}_{r, 0}^{g}=2^{1-2 r}(2 r+1) c_{2 r, 0}^{g}+2^{-1-2 r} c_{2 r+1,1}^{g}, \\
& \hat{b}_{r, 1}^{g}=2^{-2 r}(2 r+1) c_{2 r, 1}^{g}+2^{2-2 r} 2 r(2 r+1) c_{2 r-1,0}^{g},
\end{aligned}
$$

и в случае общего $s$ получаются $2^{s}$ членов $c_{\vec{k}, \vec{\alpha}}^{(g)}$ со всеми допустимыми подстановками $\left(k_{i}, 1\right) \leftrightarrow\left(k_{i}-1,0\right)$

\section{5. ТОПОЛОГИЧЕСКАЯ РЕКУРСИЯ}

В этом разделе описываются основные составляющие метода топологической рекурсии, развитого в работах [4], [5], [24], [25]. Параллельно представлению основ этого метода, общая конструкция будет конкретизирована на примере модели гауссовых средних $W_{s}^{(g)}\left(x_{1}, \ldots, x_{s}\right)$ :

1. Исходные данные включают в себя спектральную кривую $\Sigma_{x, y}=0$ с заданными на ней двумя мероморфными дифференциалами $d x$ и $d y$. Нули дифференциала $d x$ задают точки ветвления. В случае гауссовых средних эта кривая представляет собой сферу $y x-y^{2}=1$ и удобной локальной координатой на сфере оказывается функция

$$
x=e^{\lambda}+e^{-\lambda}, \quad y=e^{\lambda}, \quad d x=\left(e^{\lambda}-e^{-\lambda}\right) d \lambda .
$$

В случае гауссовых средних следует рассмотреть накрытие этой сферы двумя картами: $y=e^{\lambda}$ и $\bar{y}=e^{-\lambda}$; сферу при этом удобно представить в виде цилиндра, получаемого из полосы $\operatorname{Im} \lambda \in[0,2 \pi]$ отождествлением точек $(x, 0)$ вещественной прямой $\operatorname{Im} \lambda=0$ с точками $(x, 2 i \pi)$ прямой $\operatorname{Im} \lambda=2 \pi$. Имеем две точки ветвления $\lambda=0, i \pi$.

2. Следующая составляющая - это бергмановский 2-дифференциал $B(p, q)$, который есть симметричный дифференциал с нулевыми $A$-циклами (эти циклы отсутствуют в рассматриваемом случае кривой рода 0) и с двойным полюсом при совпадающих $p$ и $q$. Также понадобится первообразная дифференциала Бергмана $E(p, q)$, представляющая собой 1-дифференциал по $p$ и функцию от $q$, определенную соотношением $\int_{\bar{q}}^{q} B(p, \bullet)$. В случае гауссовых средних имеем

$$
B(p, q)=\frac{d e^{\lambda} d e^{\mu}}{\left(e^{\lambda}-e^{\mu}\right)^{2}}, \quad E(p, q)=\frac{d e^{\lambda}}{e^{\lambda}-e^{\mu}}, \quad p=e^{\lambda}, \quad q=e^{\mu} .
$$




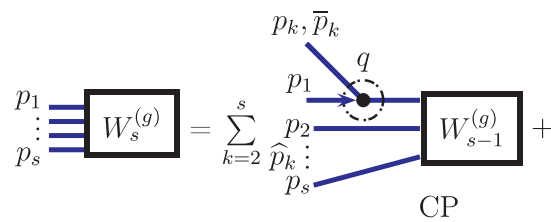

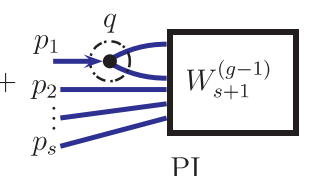

PI

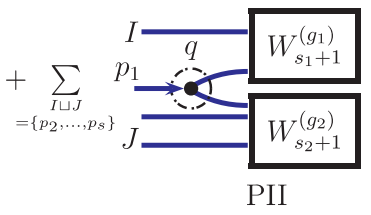

PII

Рис. 4. Графическое представление рекуррентного соотношения (5.6). Явно представлены операции копроизведения (первый член в правой части) и произведения (второй и третий члены в правой части); линии со стрелкой обозначают ядро рекурсии $K\left(p_{1}, q\right)$, а линии без стрелок отвечают ядрам Бергмана $B\left(p_{k}, q\right)$ и $B\left(\bar{p}_{k}, q\right)$.

3. Определим ядро рекурсии $K(p, q)$ как $(1,-1)$-дифференциал

$$
K(p, q)=E(p, q) \frac{1}{(y(q)-\bar{y}(q)) d x} ;
$$

для гауссовых средних имеем

$$
K(p, q)=\frac{d e^{\lambda}}{e^{\lambda}-e^{\mu}} \frac{1}{\left(e^{\mu}-e^{-\mu}\right)^{2} d \mu}, \quad p=e^{\lambda}, \quad q=e^{\mu},
$$

где один множитель $e^{\mu}-e^{-\mu}$ в знаменателе появляется из разности $y(q)-\bar{y}(q)$, а второй возникает из $d x$.

4. Введем корреляционные функции $W_{s}^{(g)}\left(p_{1}, \ldots, p_{s}\right)$ как симметричные $s$-дифференциалы, определяемые рекуррентными соотношениями: возьмем одну из переменных $\left(p_{r}\right)$ в качестве корня. Тогда

$$
\begin{gathered}
W_{3}^{(0)}\left(p_{1}, p_{2}, p_{3}\right)=\sum_{\operatorname{Res} d x=0} K\left(p_{1}, q\right)\left[B\left(p_{2}, q\right)+B\left(\bar{p}_{2}, q\right)\right]\left[B\left(p_{3}, q\right)+B\left(\bar{p}_{3}, q\right)\right], \\
W_{1}^{(1)}\left(p_{1}\right)=\sum_{\operatorname{Res} d x=0} K\left(p_{1}, q\right) B(q, \bar{q})
\end{gathered}
$$

$$
\begin{aligned}
& W^{(g)}\left(p_{1}, p_{2}, \ldots, p_{s}\right)= \\
& =\sum_{\operatorname{Res} d x=0} K\left(p_{1}, q\right)\left[\sum_{k=2}^{s}\left[B\left(p_{k}, q\right)+B\left(\bar{p}_{k}, q\right)\right] W_{s-1}^{(g)}\left(q, p_{2}, \ldots, \widehat{p}_{k}, \ldots, p_{s}\right)+\right. \\
& \left.\quad+W_{s+1}^{(g-1)}\left(q, q, p_{2}, \ldots, p_{s}\right)+\sum_{\substack{g_{1}+g_{2}=g \\
I \sqcup J=\left\{p_{2}, \ldots, p_{s}\right\}}}^{\prime} W_{|I|+1}^{\left(g_{1}\right)}\left(q,\left\{p_{i}\right\}_{i \in I}\right) W_{|J|+1}^{\left(g_{2}\right)}\left(q,\left\{p_{j}\right\}_{j \in J}\right)\right],
\end{aligned}
$$

где правая часть соотношения явно симметризована относительно всех $p_{2}, \ldots, p_{s}$, но не относительно $p_{1}$, символ $\sum^{\prime}$ означает, что в сумму дают вклад только стабильные члены (т. е. те, для которых $2 g-2+s>0$ ), и явно выделен вклад от единственной нестабильной кривой (член с $\left.\left[B\left(p_{k}, q\right)+B\left(\bar{p}_{k}, q\right)\right]\right)$. Крышка над символом обозначает его исключение из списка аргументов, а в последнем члене берется сумма по всем разбиениям множества аргументов $\left\{p_{2}, \ldots, p_{s}\right\}$ в два непересекающихся подмножества $I$ и $J$. Рекуррентное соотношение схематически изображено на рис. 4. 
С помощью рекуррентного соотношения (5.6) можно построить все старшие $W_{s}^{(g)}$ начиная с $W_{3}^{(0)}\left(p_{1}, p_{2}, p_{3}\right)$ и $W_{1}^{(1)}\left(p_{1}\right)$.

Лемма из работы [4] утверждает, что, хотя рекуррентные соотношения рекурсии (5.6) не являются явно симметричными относительно перестановок всех $p_{1}, \ldots, p_{s}$, полная сумма в правой части соотношения оказывается полностью симметричной.

5.1. Топологическая рекурсия для гауссовых средних. В любой локальной теории, подчиняющейся топологической рекурсии, все стабильные корреляционные функции $W_{s}^{(g)}\left(x_{1}, \ldots, x_{s}\right)$ имеют сингулярности только в точках ветвления. В гауссовом случае можно, тем самым, заключить, что единственными сингулярностями в правой части (5.6), кроме полюсов высоких порядков в точках ветвления (для $W_{s}^{(g)}$ максимально возможный порядок полюса равен $6 g+2 s-3$ ), оказываются простые полюсы при $q=p_{1}$, возникающие из $K\left(p_{1}, q\right)$, и двойные полюсы при $q=p_{k}, q=\bar{p}_{k}$, возникающие из $\left[B\left(p_{k}, q\right)+B\left(\bar{p}_{k}, q\right)\right]$. Тем самым, интегрирование по $q$ в правой части можно произвести, вычисляя вычеты в этих точках вместо точек ветвления, что существенно упрощает реальные вычисления.

ОПРЕДЕЛЕНИЕ 1. Для произвольной (стабильной) пары $(g, s)$ введем следующее множество допустимых диаграмм Юнга $\left(D_{1}, D_{2}\right)$ (как обычно $l(D)$ и $|D|$ обозначают соответствующую длину (число столбцов) и объем (число клеток) диаграммы Юнга $D)$ :

$$
\begin{gathered}
Y_{s}^{(g)}=\left\{\left(D_{0}, D_{1}\right)\right\}: s=l\left(D_{0}\right)+l\left(D_{1}\right), \quad D_{1} \in 2 \mathbb{Z}_{\geqslant 0}, \\
2 g-1+s+\frac{l\left(D_{1}\right)}{2} \leqslant\left|D_{0}\right|+\left|D_{1}\right| \leqslant 3 g+2 s-3 .
\end{gathered}
$$

Для каждой пары $(\vec{k}, \vec{\beta})$ определим пару $\left(D_{0}, D_{1}\right) \in Y_{s}^{(g)}$ следующим образом: разобьем множество индексов $\vec{k}$ на два непересекающихся подмножества: одно с $\beta_{i}=0$ и другое с $\beta_{i}=1$, и упорядочим $k_{i}$ внутри каждого подмножества в невозрастающем порядке, получив тем самым две диаграммы Юнга $D_{0}$ и $D_{1}$ со столбцами высоты $k_{i}$.

Диаграмма Юнга $D_{0}$ состоит из $l\left(D_{0}\right)$ столбцов положительных целых высот $t_{r}$ таких, что $t_{r}>t_{s}$ при $r<s$, обозначим через $d_{r}$ числа столбцов одинаковой высоты $t_{r}$, так что $\left|D_{0}\right|=\sum_{r} d_{r} t_{r}$. Соответственно, $D_{1}$ состоит из четного числа $l\left(D_{1}\right)$ столбцов высот $r_{j}, r_{j}>r_{i}$ при $j<i$, и обозначим через $k_{j}$ число столбцов одной и той же высоты $r_{j}$, так что $\left|D_{1}\right|=\sum_{j} k_{j} r_{j}$.

ОПРЕДЕЛЕНИЕ 2. Определим гомоморфизм

$$
\mathcal{F}: \mathbb{Z}\left[Y_{s}^{(g)}\right] \rightarrow R\left[e^{\lambda_{1}}, \ldots, e^{\lambda_{s}}\right]
$$

в пространство $R$ рациональных функций от $e^{\lambda_{i}}, i=1, \ldots, s$, рассматриваемых как формальные комплексные переменные, задаваемый отображением каждой пары $\left(D_{0}, D_{1}\right) \in Y_{s}^{(g)}$ в функцию от $\lambda_{i}, i=1, \ldots, s$, заданную формулой

$$
\mathcal{F}\left(D_{0}, D_{1}\right):=\sum_{\substack{L_{1} \sqcup L_{2} \sqcup \ldots \sqcup R_{1} \sqcup R_{2} \sqcup \cdots=\{1, \ldots, s\} \\\left|L_{i}\right|=d_{i},\left|R_{j}\right|=k_{j}}} \prod_{i}\left(\prod_{\alpha \in L_{i}} s_{t_{i}-1,0}\left(\lambda_{\alpha}\right)\right) \prod_{j}\left(\prod_{\gamma \in R_{j}} s_{r_{j}-1,1}\left(\lambda_{\gamma}\right)\right),
$$


в которой суммирование идет по всем разбиениям множества индексов $\{1, \ldots, s\}$ на непересекающиеся подмножества $R_{i}, L_{j}$ соответствующих размеров $d_{i}, k_{j}$ и $s_{k, \beta}(\lambda):=\left(e^{\lambda}+e^{-\lambda}\right)^{\beta}\left(e^{\lambda}-e^{-\lambda}\right)^{-2 k-3}$.

Теорема 6. Гауссовъ средние $W_{s}^{(g)}\left(x_{1}, \ldots, x_{s}\right)$ суть коэффициенты дифберенииалов

$$
W_{s}^{(g)}\left(p_{1}, p_{2}, \ldots, p_{s}\right)=W_{s}^{(g)}\left(x_{1}, \ldots, x_{s}\right) d x_{1} \cdots d x_{s},
$$

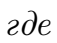

$$
\begin{gathered}
W_{s}^{(g)}\left(x_{1}, \ldots, x_{s}\right)=W_{s}^{(g)}\left(e^{\lambda_{1}}+e^{-\lambda_{1}}, \ldots, e^{\lambda_{s}}+e^{-\lambda_{s}}\right)= \\
=\sum_{\left(D_{0}, D_{1}\right) \in Y_{s}^{(g)}} \hat{b}_{D_{0}, D_{1}}^{(g)} \mathcal{F}\left(D_{0}, D_{1}\right),
\end{gathered}
$$

и коэффициенты разложения $\hat{b}_{D_{0}, D_{1}}^{(g)}$ занумерованы парами приведенных выше диаграмм Юнга $D_{0}$ и $D_{1}$.

ДоказАтельство. Рекуррентные соотношения, представленные формулой (5.6) (или в графическом виде на рис. 4), определяются двумя операциями на множестве базисных функций $s_{k, 0}(\lambda)$ и $s_{k, 1}(\lambda)$ : операциями “произведения" и "копроизведения". В п. 5.2 эти операции будут описаны непосредственно на уровне диаграмм Юнга.

Операция произведения имеет место во втором и третьем членах в (5.6): эта операция задается на множестве базисных функций и продолжается по билинейности на произведения таких функций, составляющие $W_{s}^{(g)}\left(e^{\lambda_{1}}+e^{-\lambda_{1}}, \ldots, e^{\lambda_{s}}+e^{-\lambda_{s}}\right)$. В результате этой операции из двух базисных функций $s_{k_{1}, \beta_{1}}(\lambda)$ и $s_{k_{2}, \beta_{2}}(\lambda)$ производится линейная комбинация базисных функций $s_{k, \beta}\left(\lambda_{1}\right)$, обозначаемая стандартным символом “спаривания" и определяемая следующим интегралом:

"произведение": $\quad \underbrace{}_{k_{1}, \beta_{1}}(\lambda) s_{k_{2}, \beta_{2}}(\lambda):=\sum_{\operatorname{Res} d x_{q}=0} K\left(p_{1}, q\right) s_{k_{1}, \beta_{1}}\left(\lambda_{q}\right) s_{k_{2}, \beta_{2}}\left(\lambda_{q}\right) d x_{q}^{2}$.

С учетом того, что $d x_{q}=\left(e^{\lambda_{q}}-e^{-\lambda_{q}}\right) d \lambda_{q}$ и что вместо вычисления этого интеграла по вычетам в точках ветвления его можно вычислить, взяв вычет в его единственном простом полюсе $p_{1}=q$ вне точек ветвления, получим с учетом явного вида (3.18) базисных векторов

$$
\begin{aligned}
& \sum_{\operatorname{Res} d x_{q}=0} K\left(p_{1}, q\right) s_{k_{1}, \beta_{1}}\left(\lambda_{q}\right) s_{k_{2}, \beta_{2}}\left(\lambda_{q}\right) d x_{q}^{2}=-\operatorname{Res}_{p_{1}=q} K\left(p_{1}, q\right) s_{k_{1}, \beta_{1}}\left(\lambda_{q}\right) s_{k_{2}, \beta_{2}}\left(\lambda_{q}\right) d x_{q}^{2} \\
& =\frac{\left(e^{\lambda_{1}}+e^{-\lambda_{1}}\right)^{\beta_{1}+\beta_{2}}}{\left(e^{\lambda_{1}}-e^{-\lambda_{1}}\right)^{6+2 k_{1}+2 k_{2}}} d \lambda_{1}=\frac{\left(e^{\lambda_{1}}+e^{-\lambda_{1}}\right)^{\beta_{1}+\beta_{2}}}{\left(e^{\lambda_{1}}-e^{-\lambda_{1}}\right)^{7+2 k_{1}+2 k_{2}}} d x_{1}
\end{aligned}
$$

так что, поскольку $\left(e^{\mu}+e^{-\mu}\right)^{2}=\left(e^{\mu}-e^{-\mu}\right)^{2}+4$, приходим к следующему правилу для операции произведения:

$$
\underline{s_{k_{1}, \beta_{1}}(\lambda) s_{k_{2}, \beta_{2}}(\lambda)}= \begin{cases}s_{k_{1}+k_{2}+2, \beta_{1}+\beta_{2}}\left(\lambda_{1}\right), & \beta_{1}+\beta_{2}<2, \\ s_{k_{1}+k_{2}+1,0}\left(\lambda_{1}\right)+4 s_{k_{1}+k_{2}+2,0}\left(\lambda_{1}\right), & \beta_{1}=\beta_{2}=1 .\end{cases}
$$

Вторая необходимая операция - это операция "копроизведения", встречающаяся в первом члене в правой части соотношения (5.6). Она производит из базисной функции $s_{k, \beta}(\lambda)$ член, билинейный по $s_{k_{1}, \beta_{1}}\left(\lambda_{1}\right)$ и $s_{k_{2}, \beta_{2}}\left(\lambda_{p}\right)$. Эта операция 
обозначается символом поднятия сбоку от значка этой функции, продолжается по линейности на произведение таких базисных функций и определяется следующим интегралом:

" копроизведение ": $\quad\left(s_{k, \beta}(\lambda)\right)^{\uparrow}:=\sum_{\operatorname{Res} d x_{q}=0} K\left(p_{1}, q\right)\left[B\left(p_{k}, q\right)+B\left(\bar{p}_{k}, q\right)\right] s_{k, \beta}\left(\lambda_{q}\right) d x_{q}$,

где снова интегрирование производится с помощью взятия вычетов в точках $q=p_{1}$ и $q=p_{k}$ (в члене с $\left.B\left(p_{k}, q\right)\right)$ и в точках $q=p_{1}$ и $q=\bar{p}_{k}$ (для члена с $\left.B\left(\bar{p}_{k}, q\right)\right)$. Вычисления состоят в применении комбинаторики геометрической прогрессии, но в остальном оказываются вполне стандартными. Случаи $\beta=0$ и $\beta=1$ значительно различаются, так что два интегрирования определяют два типа операции копроизведения:

$$
\begin{aligned}
& \left(s_{k, 0}(\lambda)\right)^{\uparrow}=\sum_{\operatorname{Res} d x_{q}=0} K\left(p_{1}, q\right)\left[B\left(p_{k}, q\right)+B\left(\bar{p}_{k}, q\right)\right] s_{k, 1}\left(\lambda_{q}\right) d x_{q}= \\
& =\sum_{m=0}^{k}(2+2 k-2 m) s_{m, 0}\left(\lambda_{1}\right) s_{k-m, 0}\left(\lambda_{p}\right) d x_{1} d x_{p}+ \\
& \quad+\sum_{m=0}^{k+1}(3+2 k-2 m)\left[4 s_{m, 0}\left(\lambda_{1}\right) s_{k+1-m, 0}\left(\lambda_{p}\right)+s_{m, 1}\left(\lambda_{1}\right) s_{k+1-m, 1}\left(\lambda_{p}\right)\right] d x_{1} d x_{p}
\end{aligned}
$$

и

$$
\begin{aligned}
& \left(s_{k, 1}(\lambda)\right)^{\uparrow}=\sum_{\operatorname{Res} d x_{q}=0} K\left(p_{1}, q\right)\left[B\left(p_{k}, q\right)+B\left(\bar{p}_{k}, q\right)\right] s_{k, 1}\left(\lambda_{q}\right) d x_{q}= \\
& =\sum_{m=0}^{k}(2+2 k-2 m) s_{m, 1}\left(\lambda_{1}\right) s_{k-m, 0}\left(\lambda_{p}\right) d x_{1} d x_{p}+ \\
& \quad+\sum_{m=0}^{k}(1+2 k-2 m) s_{m, 0}\left(\lambda_{1}\right) s_{k-m, 1}\left(\lambda_{p}\right) d x_{1} d x_{p}+ \\
& \quad+\sum_{m=0}^{k+1} 4(3+2 k-2 m)\left[s_{m, 0}\left(\lambda_{1}\right) s_{k+1-m, 1}\left(\lambda_{p}\right)+s_{m, 1}\left(\lambda_{1}\right) s_{k+1-m, 0}\left(\lambda_{p}\right)\right] d x_{1} d x_{p} .
\end{aligned}
$$

Две корреляционные функции, необходимые для запуска процедуры рекурсии, имеют вид

$$
\begin{aligned}
& W_{3}^{(0)}\left(x_{1}, x_{2}, x_{3}\right)=4 s_{0,0}\left(\lambda_{1}\right) s_{0,0}\left(\lambda_{2}\right) s_{0,0}\left(\lambda_{3}\right) d x_{1} d x_{2} d x_{3}+ \\
& \quad+\left[s_{0,1}\left(\lambda_{1}\right) s_{0,1}\left(\lambda_{2}\right) s_{0,0}\left(\lambda_{3}\right)+s_{0,1}\left(\lambda_{1}\right) s_{0,0}\left(\lambda_{2}\right) s_{0,1}\left(\lambda_{3}\right)+\right. \\
& \left.\quad+s_{0,0}\left(\lambda_{1}\right) s_{0,1}\left(\lambda_{2}\right) s_{0,1}\left(\lambda_{3}\right)\right] d x_{1} d x_{2} d x_{3}
\end{aligned}
$$

и

$$
W_{1}^{(1)}(x)=s_{1,0}(\lambda) d x
$$


5.2. Соотношения рекурсии, задающие $\hat{b}_{D_{0}, D_{1}}^{(g)}$. Выпишем теперь рекуррентные соотношения на коэффициенты $\hat{b}_{D_{0}, D_{1}}^{(g)}$ исключительно в терминах опера-

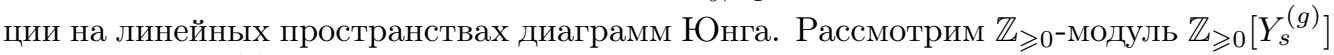
функций на $Y_{s}^{(g)}$ с неотрицательными целыми коэффициентами.

Следующая теорема представляет собой основной результат данного раздела.

Теорема 7. Все коэффициенты $\hat{b}_{D_{0}, D_{1}}^{(g)}$ разложений петлевых средних в теореме 6 в диапазоне, определяемом условиями (5.7), суть положительные целье числа, т.е. имеются однозначно определенные элементы $\mathcal{W}_{s}^{(g)} \in \mathbb{Z}_{\geqslant 0}\left[Y_{s}^{(g)}\right]$ такие, что

$$
\mathcal{F}\left(\mathcal{W}_{s}^{(g)}\right)=W_{s}^{(g)}\left(e^{\lambda_{1}}+e^{-\lambda_{1}}, \ldots, e^{\lambda_{s}}+e^{-\lambda_{s}}\right)
$$

Элемент $\mathcal{W}_{s}^{(g)} \in \mathbb{Z}_{\geqslant 0}\left[Y_{s}^{(g)}\right]$ задается рекуррентным соотношением, основанным на операциях копроизведения и произведения, описанных выше.

ДоКАЗАТЕЛЬСТВО имеет констуктивную природу и состоит в поднятии операций произведения и копроизведения на множество диаграмм Юнга. Определим сначала вспомогательное пространство диаграмм Юнга.

ОПРЕдЕлЕНиЕ 3. Зададим множество пар диаграмм Юнга $\widetilde{Y}_{s}^{(g)}$, элементы которого совпадают с элементами множества $Y_{s}^{(g)}$, снабженными следующими дополнительными структурами. Присвоим индекс 1 в точности одному из столбцов каждой пары $\left(D_{0}, D_{1}\right) \in Y_{s}^{(g)}$ (это присвоение соответствует симметризации по всем аргументам, кроме $\lambda_{1}$ в $\left.(5.8)\right)$. Элементы $\left(\widetilde{D_{0}, D_{1}}\right)$ множества $\widetilde{Y}_{s}^{(g)}$ с одними и теми же диаграммами $\left(D_{0}, D_{1}\right)$, но с присвоением индекса 1 столбцам разных "сортов" (различающихся высотами и/или "раскрасками": диаграмма $D_{0}$ раскрашена белым цветом, а диаграмма $D_{1}-$ серым) считаются различными. Рассмотрим $\mathbb{Z}_{\geqslant 0}$-модуль $\mathbb{Z}_{\geqslant 0}\left[\widetilde{Y}_{s}^{(g)}\right]$ функций на $\tilde{Y}_{s}^{(g)}$ с неотрицательными целыми коэффициентами.

ОПРЕДЕлЕНИЕ 4. Определим вложение $S$ модуля $\mathbb{Z}_{\geqslant 0}\left[Y_{s}^{(g)}\right]$ в модуль $\mathbb{Z}_{\geqslant 0}\left[\tilde{Y}_{s}^{(g)}\right]$, порождаемое следующим линейным отображением: образ элемента $S\left(D_{0}, D_{1}\right)$ есть сумма элементов $\left(\widehat{D_{0}, D_{1}}\right)$ (с единичными коэффициентами), основанных на указанной паре диаграмм Юнга $\left(D_{0}, D_{1}\right)$ и содержащих индексы 1 всех возможных сортов (см. примеры ниже). Относительно гомоморфизма $\mathcal{F}$ такие суммы становятся выражениями, полностью симметричными по всем своим $\lambda$-аргументам.

Операции произведения и копроизведения отображают модуль $\mathbb{Z}_{\geqslant 0}\left[Y_{s}^{(g)}\right]$ в модуль $\mathbb{Z}_{\geqslant 0}\left[\tilde{Y}_{s}^{(g)}\right]$. Для представления этих операций будем применять удобную графическую форму записи (как обычно, для сокращения записи будем опускать одну из диаграмм, если она пуста); первая (белая) диаграмма будет обозначать $D_{0}$, а вторая (серая) $-D_{1}$, например, $\mathcal{W}_{3}^{(0)}=4+1 \quad$ и $\mathcal{W}_{1}^{(1)}=1 \square$, а коэффициенты пар "белых" и "серых" диаграмм в точности равны $\hat{b}_{D_{0}, D_{1}}^{(g)}$.

Операция копроизведения $\mathrm{CP}: \mathbb{Z}_{\geqslant 0}\left[Y_{s}^{(g)}\right] \rightarrow \mathbb{Z}_{\geqslant 0}\left[\widetilde{Y}_{s+1}^{(g)}\right]$.

Эта операция производит два столбца (с индексами 1 и $p$ ) из столбца каждого сорта в $\left(D_{0}, D_{1}\right) \in Y_{s}^{(g)}$ в соответствии со следующими правилами. 
- Операция копроизведения применяется ровно к одному столбцу каждого сорта (сорта различаются высотой и раскраской столбцов); эта операция базируется на соотношениях (5.12) и (5.13) и записывается графически в виде

$$
\begin{aligned}
& (\mathrm{B} \uparrow k+1)^{\uparrow}=\sum_{m=0}^{k} 2(k-m+1)+m+1 \uparrow \operatorname{1p}_{1 p}^{\mathrm{B}} \uparrow^{k-m+}+ \\
& +\sum_{m=0}^{k+1}(2 k-2 m+3)[4 m+1 \uparrow \underbrace{\mathrm{B}}_{1 p}\rfloor^{k-m+2}+m+1 \uparrow \underbrace{}_{1} \mathrm{~B}_{p} \uparrow k-m+2+] \text {, } \\
& \left.(\mathrm{B} \uparrow k+1)^{\uparrow}=\sum_{m=0}^{k} 2(k-m+1) m+1 \uparrow \square_{1 p}^{\square}\right\rfloor^{\square} k-m++
\end{aligned}
$$

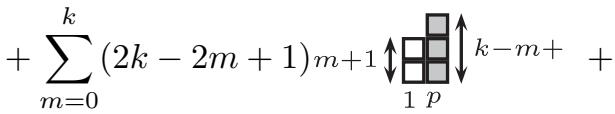

$$
\begin{aligned}
& \left.+\sum_{m=0}^{k+1} 4(2 k-2 m+3)[m+1 \uparrow \underbrace{\mathrm{B}}_{1 p} \downarrow^{k-m+2}+m+1 \uparrow \underbrace{\mathrm{B}}_{1 p}\rfloor^{k-m+2}\right] \text {. }
\end{aligned}
$$

- Получаемые таким образом столбцы с индексами 1 и $p$ встраиваются в пары диаграмм Юнга (прочие столбцы не изменяются); среди остающихся столбцов имеется $k \geqslant 0$ столбцов того же сорта, что и столбец с индексом $p$; получаемую при этом диаграмму следует умножить на $k+1$ и стереть индекс $p$, но оставить индекс 1.

Поэтому в результате применения операции копроизведения возникает линейная комбинация пар диаграмм Юнга с положительными целыми коэффициентами. Ровно один столбец в каждой паре несет индекс 1.

Пример 3. Вычислим сначала $\mathcal{W}_{4}^{(0)}$. Так как в этом случае операция произведения не дает вклада, ответ получается при действии операции копроизведения на $\mathcal{W}_{3}^{(0)}$. Для элементов этой диаграммы Юнга имеем

$$
\begin{aligned}
& ()^{\uparrow}=2{ }_{1} \cdot{ }_{p}+4 \underset{1}{\square} \cdot{ }_{p}+12 \underset{1}{ } \cdot \underset{p}{\square}+3 \underset{1}{\square} \cdot \underset{p}{\square}+1 \underset{1}{\square} \cdot{ }_{p}, \\
& ()^{\uparrow}=2 \underset{1}{ } \cdot{ }_{p}+4 \underset{1}{\square} \cdot{ }_{p}+1{ }_{1} \cdot{ }_{p}+4 \underset{1}{\square} \cdot p_{p}+12 \underset{1}{ } \cdot \underset{p}{\square}+12 \underset{1}{ } \cdot \underset{p}{\square} \text {. }
\end{aligned}
$$

Таким образом, для элементов $\mathcal{W}_{3}^{(0)}$ получаем (в выражении ниже явно выделены множители, возникающие из-за симметризации по $p$ )

$$
\begin{aligned}
& \left(\mathcal{W}_{3}^{(0)}\right)^{\uparrow}=4()^{\uparrow}+()^{\uparrow}+()^{\uparrow}= \\
& =8 \cdot 3_{1 p}+16 \cdot 3 \underset{1 p}{\square}+48 \underset{p 1}{\square}+12 \quad \underset{p 1}{\square}+4 \quad \underset{1 p}{\square}+ \\
& +2 \underset{1 p}{ }+4 \underset{1 p}{\square}+12 \square_{p 1}+3 \underset{p 1}{\square}+1 \cdot 3 \underset{1 p}{\square}+
\end{aligned}
$$

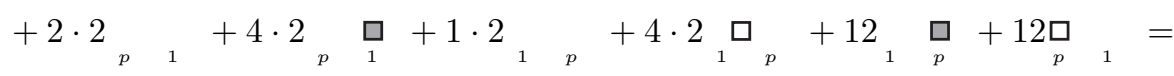

$$
\begin{aligned}
& =24_{1}+48\left(\begin{array}{ll}
\square_{1} & +\square_{1}
\end{array}\right)+12\left(\begin{array}{cc}
\square_{1} & \square_{1} \\
& +_{1}
\end{array}\right)+
\end{aligned}
$$

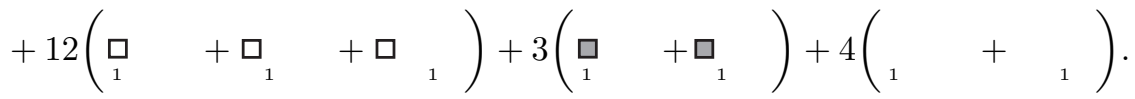


Видно, что автоматически получаются симметризованные выражения относительно $p_{1}$ : каждый член в скобках содержит ровно один столбец с индексом "1" для столбцов каждого сорта, и это выражение, таким образом, лежит в образе отображения $S$. При отображении $\mathcal{F}$ полный ответ тем самым полностью симметричен по всем своим аргументам и имеет вид

$$
\mathcal{W}_{4}^{(0)}=24 \quad+48 \square \quad+12 \quad \square+12 \square \quad+3 \square \quad+4 \quad \text {. }
$$

Тем самым получается выражение из модуля $\mathbb{Z}_{\geqslant 0}\left[Y_{4}^{(0)}\right]$. Коэффициенты этого выражения в точности равны $\hat{b}_{D_{0}, D_{1}}$ для планарного случая с четырьмя остовами.

Операция произведения производит один столбец с индексом "1" (или линейную комбинацию таких столбцов) из двух столбцов в соответствии со следующими правилами:

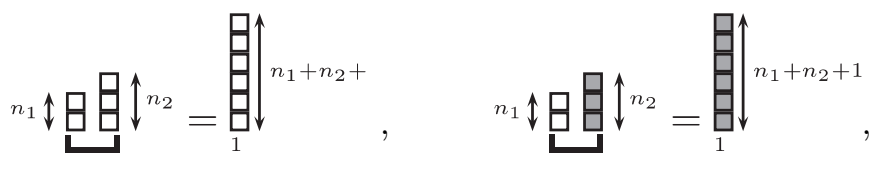

$$
\begin{aligned}
& \left.n_{1} \uparrow \mathrm{G} \uparrow^{n_{2}}=4 \underset{1}{\mathrm{G}} \rrbracket_{n_{1}+n_{2}+1}^{\mathrm{G}}+\underset{1}{\mathrm{~B}}\right\rfloor_{n_{1}+n_{2}} .
\end{aligned}
$$

Имеются два случая.

1. Первый случай имеет место, когда произведение производится внутри одной и той же пары диаграмм $\left(D_{0}, D_{1}\right)$ :

$$
\mathrm{PI}: \mathbb{Z}_{\geqslant 0}\left[Y_{s}^{(g)}\right] \rightarrow \mathbb{Z}_{\geqslant 0}\left[\tilde{Y}_{s-1}^{(g+1)}\right]
$$

В этом случае необходимо взять все возможные (попарные) произведения между различными сортами столбцов (одна операция произведения на каждую пару сортов столбцов), равно как и произведения внутри множеств столбцов одного сорта (если в $D_{0}$ или в $D_{1}$ имеется более одного столбца данного сорта). Дополнительно возникают следующие множители:

- множитель 2, если произведение берется между столбцами различных сортов;

- дополительный множитель 2, если произведение берется в члене корреляционной функции $\mathcal{W}_{s}^{(g)}$ с $s>2$, т. е. если результат действия операции PI на $\left(D_{0}, D_{1}\right)$ содержит более одного столбца.

2. Второй случай соответствует применению операции произведения к двум различным парам диаграмм Юнга $\left(D_{0}, D_{1}\right) \in Y_{s_{1}}^{\left(g_{1}\right)}$ и $\left(D_{0}^{\prime}, D_{1}^{\prime}\right) \in Y_{s_{2}}^{\left(g_{2}\right)}$ :

$$
\mathrm{PII}: \mathbb{Z}_{\geqslant 0}\left[Y_{s_{1}}^{\left(g_{1}\right)}\right] \times \mathbb{Z}_{\geqslant 0}\left[Y_{s_{2}}^{\left(g_{2}\right)}\right] \rightarrow \mathbb{Z}_{\geqslant 0}\left[\widetilde{Y}_{s_{1}+s_{2}-1}^{\left(g_{1}+g_{2}\right)}\right]
$$

В этом случае необходимо вычислить все возможные произведения между столбцами всех сортов в первой и во второй парах (одно произведение на каждую пару сортов; в каждой из двух пар диаграмм Юнга выбирается по одному сорту), если не выполняется условие $\left(D_{0}, D_{1}\right)=\left(D_{0}^{\prime}, D_{1}^{\prime}\right)$; в противном случае каждый тип спаривания между столбцами диаграммы $\left(D_{0} D_{1}\right)$ берется один раз. В итоге получается 
объединение двух приведенных выше пар диаграмм Юнга, и в результате применения операции произведения получается линейная комбинация диаграмм Юнга следующего вида:

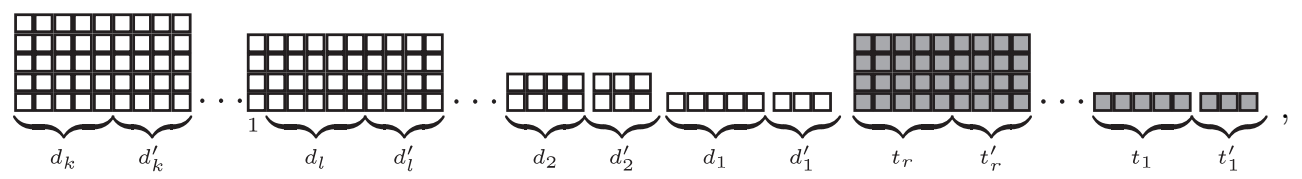

в которой в точности один столбец несет индекс 1 и в каждом члене $d_{j}$ столбцов возникает из диаграммы $D_{0}, d_{j}^{\prime}$ столбцов - из диаграммы $D_{0}^{\prime}$, и, соответственно, $t_{i}$ столбцов возникают из диаграммы $D_{1}$ и $t_{i}^{\prime}$ столбцов - из диаграммы $D_{1}^{\prime}$.

Необходимые комбинаторные множители перечислены ниже.

- Получаемая диаграмма Юнга умножается на произведение биномиальных множителей:

$$
\prod_{j=1}^{k}\left(\begin{array}{c}
d_{j}+d_{j}^{\prime} \\
d_{j}
\end{array}\right) \prod_{i=1}^{r}\left(\begin{array}{c}
t_{i}+t_{i}^{\prime} \\
t_{i}
\end{array}\right)
$$

- Имеется дополнительный множитель, равный 2, если произведение берется между столбцами различных сортов и/или между двумя различными диаграммами (т. е. если $D_{0} \neq D_{0}^{\prime}$ и/или $D_{1} \neq D_{1}^{\prime}$ ) (другими словами, единственная ситуация, в которой этот множитель отсутствует, имеет место при вычислении произведения между двумя одинаковыми диаграммами Юнга, $D_{0}=D_{0}^{\prime}$ и $D_{1}=D_{1}^{\prime}$, и только если вычисляется произведение между столбцами одного и того же сорта в этих диаграммах).

- Следует дополнительно умножить результат на 2 , если результат произведения диаграмм Юнга $\left(D_{0}, D_{1}\right)$ и $\left(D_{0}^{\prime}, D_{1}^{\prime}\right)$ содержит более одного столбца, т. е. если $s_{1}=$ $l\left(D_{0}\right)+l\left(D_{1}\right)>1$ и/или $s_{2}=l\left(D_{0}^{\prime}\right)+l\left(D_{1}^{\prime}\right)>1$ (другими словами, единственная ситуация, в которой этот множитель отсутствует, возникает при $s_{1}=s_{2}=1$ ).

ПримеР 4. Вычислим теперь $\mathcal{W}_{2}^{(1)}$ (в третьей строке явно выделены комбинаторные множители, возникающие в результате применения операции произведения):

$$
\begin{aligned}
& \mathcal{W}_{2}^{(1)}=\left(\mathcal{W}_{1}^{(1)}\right)^{\uparrow}+\mathcal{W}_{3}^{(0)}=(\square)^{\uparrow}+4 \longleftarrow+\longleftarrow+\longleftarrow= \\
& =4 \square_{1}+12 \underset{1}{\square}+2 \square_{1}+4 \underset{1}{\theta}+20 \theta_{1}+5 日_{1}+3 \underset{1}{\square}+\underset{1}{日}+ \\
& +4 \cdot 2 \underset{1}{\mathrm{G}}+1 \cdot 4 \underset{1}{\mathrm{\theta}}+4 \cdot 2 \underset{1}{\mathrm{~B}}+1 \cdot 2 \underset{1}{\mathrm{a}}=
\end{aligned}
$$

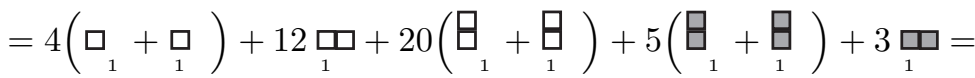

$$
\begin{aligned}
& =4 \text { ㅁ } 12 \text { 무 } 20 \text { 日 }+5 \text { 日 + } 3 \text { ㅁ. }
\end{aligned}
$$


Отсюда и из выражения для $\mathcal{W}_{1}^{(1)}$ можно вычислить $\mathcal{W}_{1}^{(2)}$ :

$$
\begin{aligned}
& \mathcal{W}_{1}^{(2)}=\underline{\mathcal{W}}_{2}^{(1)}+\underline{\mathcal{W}}_{1}^{(1)} \times \mathcal{W}_{1}^{(1)}=4 \text { 문 }+12 \text { 문 }+20 \text { 日 }+5 \text { 日 }+3 \text { 문 }+ \text { 문무 }=
\end{aligned}
$$

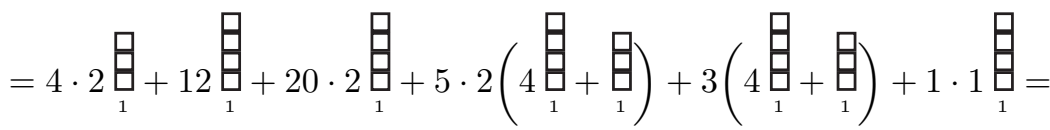

$$
\begin{aligned}
& =3 \cdot 5 \cdot 7 \text { 日 }+3 \cdot 7 \text { 日. }
\end{aligned}
$$

Такой же ответ получается при применении рекуррентной формулы Харера-Цагира: $b_{1}^{(2)}=3 \cdot 5 \cdot 7, b_{0}^{(2)}=3 \cdot 7$.

Пример 5. Первый пример, в котором дают вклад все три операции, описанные выше, - это вычисление $\mathcal{W}_{3}^{(1)}$ :

$$
\mathcal{W}_{3}^{(1)}=\left(\mathcal{W}_{2}^{(1)}\right)^{\uparrow}+\mathcal{W}_{4}^{(0)}+\mathcal{W}_{3}^{(0)} \times \mathcal{W}_{1}^{(1)} .
$$

Здесь первый член (с копроизведением) содержит 67 слагаемых, второй содержит 21 слагаемое, а третий - только три слагаемых. Выполняя суммирование, мы снова получаем ответ, полностью симметричный относительно всех $p_{i}$, включая $p_{1}$. Этот ответ имеет вид

$$
\begin{aligned}
& \mathcal{W}_{3}^{(1)}=24 \square+192 \square+240 \text { 日 }+288 \square+480 \text { 口 }+560 \text { 日 }+30 \text { 日 }+
\end{aligned}
$$

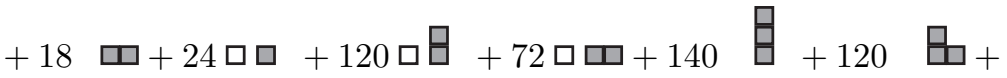

$$
\begin{aligned}
& +120 \text { 日 }+30 \text { 日 }+140 \text { 日. }
\end{aligned}
$$

Второй пример - это $\mathcal{W}_{5}^{(0)}$ :

$$
\mathcal{W}_{5}^{(0)}=\left(\mathcal{W}_{4}^{(0)}\right)^{\uparrow}+\underline{\mathcal{W}}_{3}^{(0)} \times \mathcal{W}_{3}^{(0)} .
$$

Первый член содержит 85 слагаемых, а второй - семь слагаемых, которые приводятся ниже для прояснения процедуры подсчета симметрийных множителей (единичные биномиальные множители опущены для простоты):

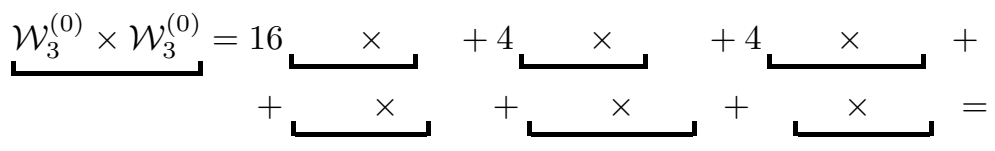

$$
\begin{aligned}
& =16 \cdot 2 \cdot\left(\begin{array}{l}
4 \\
2
\end{array}\right) \mathrm{B} \quad+4 \cdot 2 \cdot 2 \mathrm{~B} \quad+4 \cdot 2 \cdot 2 \cdot\left(\begin{array}{l}
3 \\
1
\end{array}\right) \quad \underset{1}{\mathrm{~B}}+
\end{aligned}
$$

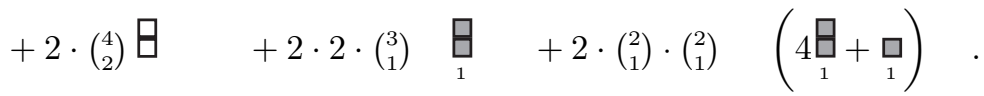


Поскольку здесь операция произведения применяется к идентичным объектам, каждый тип произведения учитывается только один раз, но если вычисляется произведение между различными столбцами для столбцов одного и того же сорта, то необходимо умножить на 2. Один множитель, равный 2, всегда присутствует, так как результат произведения содержит более одного столбца.

Сумма в выражении (5.26) оказывается полностью симметричной относительно всех $p_{i}$, включая $p_{1}$, и мы получаем ответ:

$$
\begin{aligned}
& \mathcal{W}_{5}^{(0)}=192+768 \square \quad+1152 \square \quad+960 \text { 口 } \quad+144 \quad \square+ \\
& +240 \text { 日 }+288 \square \quad \square+288 \quad \square+144 \square \quad+288 \square \quad+ \\
& +240 \text { 日 }+24 \quad+72 \text { 口 }+60 \text { 日 }+18 \text { 口 + } \\
& +72 \text { 口 } \quad+12 \text { 口 } \quad+60 \text { 日 }
\end{aligned}
$$

Очевидно, что все коэффициенты в соотношениях произведения и копроизведения представляют собой положительные целые числа, так что результат всегда целый и положительный. Более того, из леммы работы [4] следует, что результат совместного применения операций копроизведения и произведения автоматически оказывается симметричным относительно перестановок всех аргументов, включая $p_{1}$, так что результат совместного применения операций CP, PI, и РІІ принадлежит образу модуля $S\left(Y_{s}^{(g)}\right)$ в модуле $\widetilde{Y}_{s}^{(g)}$, который тем самым естественно отождествляется с собственно модулем $Y_{(g)_{s}}$ :

$$
\mathrm{CP}\left(\mathcal{W}_{s-1}^{(g)}\right)+\operatorname{PI}\left(\mathcal{W}_{s+1}^{(g-1)}\right)+\sum_{\substack{g_{1}+g_{2}=g \\ s_{1}+s_{2}=s+1}} \operatorname{PII}\left(\mathcal{W}_{s_{1}}^{\left(g_{1}\right)} \times \mathcal{W}_{s_{2}}^{\left(g_{2}\right)}\right)=\mathcal{W}_{s}^{(g)} \in \mathbb{Z}_{\geqslant 0}\left[Y_{s}^{(g)}\right]
$$

Это завершает доказательство теоремы.

\section{6. СЛУЧАЙ ОДНОГО ОСТОВА}

\section{1. Рекуррентное соотношение Харера-Цагира и представление в тер-} минах графов из раздела 3. В случае хордовых диаграмм с одним остовом имеются представление (3.13) и альтернативное представление

$$
\begin{aligned}
& W_{1}^{(g)}\left(e^{\lambda}+e^{-\lambda}\right)= \\
& =\sum_{r=0}^{3 g-2}(-1)^{r} \frac{\varkappa_{g, 1, r}}{2^{d-r}(d-r) !} \frac{1}{e^{\lambda}-e^{-\lambda}}\left(\frac{\partial}{\partial \lambda}\right)^{2 d-2 r+1} \frac{2}{e^{2 \lambda}-1}, \quad d=3 g-2,
\end{aligned}
$$

где из рассуждений, связанных со стратификацией замкнутых пространств модулей, следует, что $\varkappa_{g, 1, r}$ - гипотетически положительные рациональные числа, $\varkappa_{g, 1,0}=$ $\left\langle\tau_{3 g-2}\right\rangle_{g}$.

Для коэффициентов $b_{i}^{(g)}$ из разложения (3.13) на основе рекуррентной формулы Харера-Цагира [1] нами было получено следующее рекуррентное соотношение (независимо найденное в работе [42]). 
ПреДЛОЖЕНИЕ 1 [9]. Коэфбициентъ $b_{k}^{(g)}$ разложения (3.13) удовлетворяют трехчленному рекуррентному соотношению

$$
\begin{aligned}
(4 g+2 k+6) b_{k}^{(g+1)}= & (4 g+2 k+1)(4 g+2 k+3)\left[(4 g+2 k+2) b_{k}^{(g)}+\right. \\
& \left.+4(4 g+2 k-1) b_{k-1}^{(g)}\right] .
\end{aligned}
$$

Все эти коэффициенты - положительные целые числа ${ }^{1)}$.

В работе [9] с помощью рекуррентного соотношения (6.2) был развит метод, позволяющий определить $b_{g-1-k}^{(g)}$ при каждом фиксированном $k \geqslant 0$ для всех родов $g$. Например, для граничных коэффициентов имеются всего лишь двухчленные соотношения:

$$
\begin{aligned}
& (4 g+6) b_{0}^{(g+1)}=(4 g-1)(4 g+3)(4 g+2) b_{0}^{(g)}, \\
& (6 g+6) b_{g}^{(g+1)}=4(6 g+1)(6 g+3)(6 g-1) b_{g-1}^{(g)},
\end{aligned}
$$

из которых немедленно следует, что

$$
b_{g-1}^{(g)}=\frac{2^{g-1}(6 g-3) ! !}{3^{g} g !}, \quad b_{0}^{(g)}=\frac{(4 g) !}{8^{g} g !(2 g+1) ! !} .
$$

Подставив $b_{g-1}^{(g)}$ в соотношение $(6.1)$ и вычислив ведущий член $(r=0)$, получим, что старший коэффициент Концевича имеет вид $\varkappa_{g, 1,0}=\left\langle\tau_{3 g-2}\right\rangle_{g}=1 / 2^{3 g} 3^{g} g$ !.

Решение рекуррентного соотношения (6.2) для первого поправочного члена имеет вид

$$
b_{g-2}^{(g)}=\frac{1}{5} \frac{2^{g-2}(6 g-5) ! !}{3^{g-2}(g-2) !}, \quad \text { или } \quad \varkappa_{g, 1,1}=\frac{1}{5}\left[12 g^{2}-7 g+5\right] \varkappa_{g, 1,0}, \quad g \geqslant 2 .
$$

Для следующего члена получим

$$
b_{g-3}^{(g)}=\frac{(2 g-1) 2^{g-3}(6 g-7) ! !}{5^{2} 3^{g-3}(g-3) !}-\frac{72^{g-3}(6 g-7) ! !}{10(3 g-2) ! ! !}, \quad(3 g-2) ! ! ! \equiv \prod_{k=3}^{g}(3 k-2),
$$

и т. д. Полная многоступенчатая процедура построения этих членов описана в работе [9].

Альтернативный вывод формулы для $b_{g-2}^{(g)}$ можно произвести с помощью графического представления из леммы 4. Для этого достаточно взять только часть с временами $T_{2 k}^{+}$. Старший член для рода $g$ есть $\left\langle\tau_{3 g-2}\right\rangle_{g} T_{6 g-4}^{+}$.

Согласно лемме 4 первый поправочный член, или коэффициент $T_{6 g-6}^{+}$, возникает только из двух членов: из графа с одной вершиной и одним внутренним ребром с маркировкой концов ребра $(0,0)$ и из графа с одной вершиной и одним полуребром с маркировкой 2 (см. рис. 5): соответствующий коэффициент имеет вид

$$
\frac{B_{2}}{4}\left\langle\tau_{3 g-3} \tau_{0} \tau_{0}\right\rangle_{g-1}+\frac{2^{3}}{5 !}\left\langle\tau_{3 g-3} \tau_{2}\right\rangle_{g}
$$

1) Разумеется, свойство положительности и целочисленности коэффициентов $b_{k}^{(g)}$ есть частный случай общего утверждения теоремы 7. 


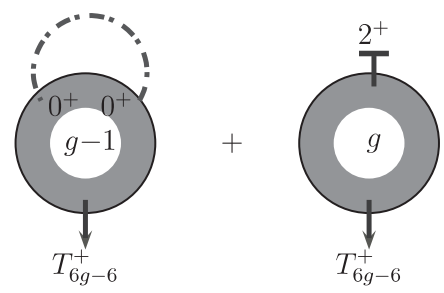

Рис. 5. Две диаграммы, дающие вклад в $b_{g-2}^{(g)}$.

и нам нужно только узнать соответствующий индекс пересечений. В то время как $\left\langle\tau_{3 g-3} \tau_{0} \tau_{0}\right\rangle_{g-1}=\left\langle\tau_{3 g-5}\right\rangle_{g-1}$, в работе [9] был вычислен индекс пересечений $\left\langle\tau_{3 g-3} \tau_{2}\right\rangle_{g}$ с применением условий Вирасоро для матричной модели Концевича; результат имеет вид

$$
\left\langle\tau_{2} \tau_{3 g-3}\right\rangle_{g}=\frac{1}{5}[12 g(g-1)+5]\left\langle\tau_{3 g-2}\right\rangle_{g}, \quad g \geqslant 2 .
$$

Применяя формулу (5.7) и тот факт, что $B_{2}=1 / 24$, получим, что коэффициент при $T_{6 g-6}^{+}$равен $\left[12 g^{2}-7 g+5\right] / 5$ в полном согласии с формулой $(6.4)$.

Ниже будет представлен третий способ вычисления той же самой величины с применением явного диаграммного счета.

\subsection{2-циклы и рекуррентные соотношения для членов $b_{g-1}^{(g)}$ и $b_{g-2}^{(g)}$.}

6.2.1. Контракция ребер в графе рода $g$. Найдем теперь коэффициент $b_{g-2}^{(g)}$ путем прямого подсчета числа ленточных графов. Для этого рассмотрим множество форм с одной граничной компонентой и одним отмеченным ребром. Обозначим через $\Gamma^{(g)}$ множество комбинаторных типов соответствующих форм рода $g$, а через $V^{(g)}-$ мощности соответствующих множеств.

Рассмотрим сначала процедуру контракции (сжатия) ребра в графах рода $g$. Через $\Gamma_{q ; 3-3}^{(g)}$ обозначим множества форм рода $g$ с отмеченным ребром таких, что все их вершины имеют валентность 3, и эти формы содержат $q$ 2-циклов (все эти 2-циклы имеют вид, изображенный на самой правой диаграмме на рис. 6). Обозначим чеpeз $V_{q ; 3-3}^{(g)}$ число таких диаграмм. Обозначим также через $\Gamma_{4,3-3}^{(g)}, \Gamma_{4,4,3-3}^{(g)}$ и $\Gamma_{5,3-3}^{(g)}$ соответственно множества форм рода $g$ с отмеченным ребром и с одной четырехвалентной вершиной, двумя четырехвалентными вершинами и одной пятивалентной вершиной в то время, как все прочие вершины имеют валентность 3. Обозначим через $V_{4,3-3}^{(g)}, V_{4,4,3-3}^{(g)}$ и $V_{5,3-3}^{(g)}$ числа соответствующих форм.

Рассмотрим теперь процесс контракции ребер. Отмеченное ребро, отвечающее концам остова, никогда не сжимается, а любое прочее ребро произвольного графа из $\Gamma_{q ; 3-3}^{(g)}$ можно сжать (тем самым имеется всего $6 g-4$ сжимаемых ребер); при этом каждый раз получается граф из $\Gamma_{4,3-3}^{(g)}$. Наоборот, каждый граф из множества $\Gamma_{4,3-3}^{(g)}$ можно получить ровно из двух графов из множества $\Gamma_{q ; 3-3}^{(g)}$; тем самым имеет место равенство

$$
(6 g-4) \sum_{q=0}^{\max } V_{q ; 3-3}^{(g)}=2 V_{4,3-3}^{(g)}=(6 g-4) b_{g-1}^{(g)} \text {. }
$$


Более интересная ситуация возникает, если сжимаются два ребра. В этом случае имеются три возможных исхода:

1) при сжатии двух непересекающихся в вершинах ребер получается граф из $V_{4,4,3-3}^{(g)}$;

2) при сжатии двух ребер, пересекающихся ровно в одной вершине, получается граф из множества $V_{5,3-3}^{(g)}$;

3) сжатие двух ребер, пересекающихся в двух вершинах (и тем самым образующих 2-петлю) невозможно.

Начнем с первого варианта. Полное число пар непересекающихся ребер равно

$$
\frac{1}{2}(6 g-4)(6 g-5)-\text { число инцидентных пар ребер. }
$$

Число пар ребер с одним или двумя пересечениями в графе из множества $V_{q, 3-3}^{(g)}$ легко подсчитать: это трижды число вершин минус четыре (в силу наличия отмеченного ребра) минус дважды число 2-петель, т. е. $3(4 g-2)-4-2 q$. Число пар ребер, пересекающихся в двух вершинах, очевидно, равно $q$. Тогда легко вычислить полное число пар непересекающихся ребер: оно равно $(3 g-4)(6 g-5)+q$. Заметим, что из каждой такой пары получается граф из множества $V_{4,4,3-3}^{(g)}$, и каждый граф из множества $V_{4,4,3-3}^{(g)}$ можно получить ровно четырьмя способами из $V_{q ; 3-3}^{(g)}$ с некоторым $q$ (при этом может случиться, что один и тот же граф из $V_{q ; 3-3}^{(g)}$ производит граф из $V_{4,4,3-3}^{(g)}$ несколькими сжатиями, этот случай будет учитываться с соответствующей кратностью). В итоге получаем соотношение

$$
\sum_{q=0}^{\max }[(3 g-4)(6 g-5)+q] V_{q ; 3-3}^{(g)}=4 V_{4,4,3-3}^{(g)} .
$$

Аналогично каждый граф из $V_{5,3-3}^{(g)}$ можно получить сжатием двух ребер с однократным пересечением ровно пятью способами из графов, принадлежащих множеству $V_{q ; 3-3}^{(g)}$, т. е. имеет место соотношение

$$
\sum_{q=0}^{\max }[12 g-10-2 q] V_{q ; 3-3}^{(g)}=5 V_{5,3-3}^{(g)} .
$$

Полное число диаграмм с $6 g-6$ немаркированными ребрами равно в точности сумме $V_{4,4,3-3}^{(g)}$ и $V_{5,3-3}^{(g)}$, и оно задается комбинацией $b$ множителей, так что получаем

$$
V_{4,4,3-3}^{(g)}+V_{5,3-3}^{(g)}=b_{g-2}^{(g)}+\frac{(3 g-2)(3 g-3)}{2} b_{g-1}^{(g)} .
$$

Тем самым имеются три уравнения, приведенные выше, на три неизвестных $V_{4,4,3-3}^{(g)}$, $V_{5,3-3}^{(g)}$ и $\sum_{q=1}^{\max } q V_{q ; 3-3}^{(g)}$. Решение имеет вид

$$
\begin{aligned}
V_{4,4,3-3}^{(g)} & =\frac{1}{4}\left[(3 g-4)(6 g-5) b_{g-1}^{(g)}+g b_{g-1}^{(g)}-\frac{20}{3} b_{g-2}^{(g)}\right], \\
V_{5,3-3}^{(g)} & =2(g-1) b_{g-1}^{(g)}+\frac{8}{3} b_{g-2}^{(g)}, \\
\sum_{q} q V_{q ; 3-3}^{(g)} & =g b_{g-1}^{(g)}-\frac{20}{3} b_{g-2}^{(g)} .
\end{aligned}
$$




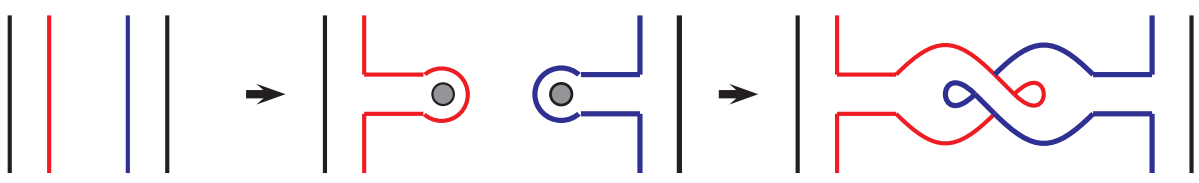

Рис. 6. Процедура вклейки ручки в две стороны двух произвольных ребер трехвалентного графа $\Gamma_{3 \ldots 3}$, увеличивающая род поверхности на единицу. Эту процедуру можно представить как раздутие ручки из пары выколотых точек.

Заметим, что еще одна комбинация величин $V$ задает интересное соотношение:

$$
(2 !) 2^{2} V_{4,4,3-3}^{(g)}+5 V_{5,3-3}^{(g)}=(6 g-5)(6 g-6) V_{3-3}^{(g)}, \quad V_{3-3}^{(g)}=\sum_{q=0}^{\max } V_{q ; 3-3}^{(g)} .
$$

Мы проверили выполнение этого соотношения для $g=3$ с использованием данных из работы [43].

6.2.2. Процесс раздутия, $g \rightarrow g+1$. Рассмотрим теперь “обратный" процесс, изображенный на рис. 6 , который позволяет раздуть ручку из пары отмеченных сторон ребер в графе из множества $\Gamma_{q ; 3-3}^{(g)}$; здесь число 2 -циклов не играет роли. На первом этапе выдуваются "пузыри", как в центральной диаграмме на рис. 6, на двух сторонах ребер; теперь раздутие пузырей возможно также и на отмеченном ребре. Также представим теперь это отмеченное ребро как подграф из трех ребер, соединенных в единственной вершине: два ребра связаны с остальной частью диаграммы (их концы суть концы отмеченного ребра, а потому всегда различны), а третье ребро представляет собой внешнюю линию. Процесс образования пузырей можно рассматривать по порядку, тогда на первой стадии имеются $2(6 g-1)$ возможностей расположить пузырь на стороне некоторого ребра, а на второй стадии уже имеется $2(6 g+1)$ таких вариантов, поскольку полное число ребер увеличивается на два после первой стадии процесса. Поэтому полное число возможностей равно

$$
\frac{1}{2} 2^{2}(6 g-1)(6 g+1)=2(6 g-1)(6 g+1) .
$$

Каждый раз, когда выдуваются два пузыря в графе $\Gamma_{3-3}^{(g)}$, в итоге получается граф из $\Gamma_{q ; 3-3}^{(g+1)}$ с $q \neq 0$. Обратно, каждый граф из $\Gamma_{q ; 3-3}^{(g+1)}$ с ненулевым $q$ можно получить ровно $q$ способами из графов из множества $\Gamma_{3-3}^{(g)}$. (Заметим, что число 2 -циклов не обязательно возрастает в ходе этого процесса: если пузырь раздувается на стороне ребра, которое само входит в некоторый 2-цикл исходного графа, то этот исходный 2-цикл разрушается в ходе процесса раздутия, так что в принципе число 2-циклов может даже уменьшаться, но каждый раз получается граф рода $g+1$ с хотя бы одним 2-циклом.)

Таким образом, получаем соотношение

$$
2(6 g-1)(6 g+1) V_{3-3}^{(g)}=\sum_{q=0}^{\max } q V_{q ; 3-3}^{(g+1)},
$$


из которого, после подстановки этого результата в (6.15) и с учетом того факта, что $V_{3-3}^{(g)}$ просто равно $b_{g-1}^{(g)}$, получаем новое соотношение на величины $b$ :

$$
2(6 g-1)(6 g+1) b_{g-1}^{(g)}=(g+1) b_{g}^{(g+1)}-\frac{20}{3} b_{g-1}^{(g+1)} .
$$

Из этого соотношения сразу получим, что

$$
b_{g-2}^{(g)}=\frac{3}{10} \frac{g(g-1)}{2 g-1} b_{g-1}^{(g)},
$$

что совпадает с формулой (6.4).

\section{7. ЗАКЛЮЧЕНИЕ}

Применение метода топологической рекурсии к построению производящих функций когомологических теорий поля занимает важное место в современной математической физике (см., например, недавнюю работу [44], в которой все инварианты полей потомков для всех порядков разложения по родам для эквивариантных инвариантов Громова-Виттена проективной прямой $\mathbb{P}^{1}$ были построены с помощью метода топологической рекурсии). В этой связи предсталяется интересным исследовать возможность применения разложения гивенталевского типа в подходе квантовой спектральной кривой.

\section{Список литературы}

[1] J. Harer, D. Zagier, Invent. Math., 85:3 (1986), 457-485.

[2] É. Brezin, S. Hikami, Commun. Math. Phys., 283:2 (2008), 507-521, arXiv: 0708.2210.

[3] A. Morozov, Sh. Shakirov, From Brezin-Hikami to Harer-Zagier formulas for Gaussian correlators, arXiv: 1007.4100.

[4] L. Chekhov, B. Eynard, JHEP, 03 (2006), 014, 18 pp., arXiv: hep-th/0504116.

[5] B. Eynard, N. Orantin, Commun. Number Theory Phys., 1:2 (2007), 347-452.

[6] S. Gukov, P. Sułkowski, JHEP, 2 (2012), 070, 56 pp., arXiv: 1108.0002.

[7] M. Mulase, P. Sułkowski, Spectral curves and the Schrödinger equations for the Eynard-Orantin recursion, arXiv: 1210.3006.

[8] O. Dumitrescu, M. Mulase, Quantization of spectral curves for meromorphic Higgs bundles through topological recursion, arXiv: 1411.1023.

[9] J.E. Andersen, L. O. Chekhov, P. Norbury, R. C. Penner, Models of discretized moduli spaces, cohomological field theories, and Gaussian means, arXiv: 1501.05867.

[10] L. Chekhov, Yu. Makeenko, Modern Phys. Lett. A, 7:14 (1992), 1223-1236, arXiv: hep-th/9201033.

[11] J. Ambjørn, L. Chekhov, C. F. Kristjansen, Yu. Makeenko, Nucl. Phys. B, 404:1-2 (1993), 127-172, arXiv: hep-th/9302014; Erratum, 449:3 (1995), 681.

[12] Y. Manin, Frobenius Manifolds, Quantum Cohomology, and Moduli Spaces, American Mathematical Society Colloquium Publications, 47, AMS, Providence, RI, 1999.

[13] L. Chekhov, Yu. Makeenko, Phys. Lett. B, 278:3 (1992), 271-278, arXiv: hep-th/9202006.

[14] A. Marshakov, A. Mironov, A. Morozov, Phys. Lett. B, 265:1-2 (1991), 99-107.

[15] P. Norbury, Quantum curves and topological recursion, arXiv: 1502.04394.

[16] L. Chekhov, J. Geom. Phys., 12:3 (1993), 153-164, arXiv: hep-th/9205106.

[17] P. Norbury, Trans. Amer. Math. Soc., 365:4 (2013), 1687-1709. 
[18] M. Mulase, M. Penkava, Adv. Math., 230:3 (2012), 1322-1339, arXiv: 1009.2135.

[19] P. Norbury, Math. Res. Lett., 17:3 (2010), 467-481.

[20] L. Chekhov, Acta Appl. Math., 48:1 (1997), 33-90, arXiv: hep-th/9509001.

[21] A. B. Givental, Mosc. Math. J., 1:4 (2001), 551-568.

[22] B. Eynard, Commun. Number Theory Phys., 8:3 (2014), 541-588, arXiv: 1110.2949.

[23] P. Dunin-Barkowski, N. Orantin, S. Shadrin, L. Spitz, Commun. Math. Phys., 328:2 (2014), 669-700, arXiv: 1211.4021.

[24] B. Eynard, JHEP, 11 (2004), 031, 35 pp., arXiv: hep-th/0407261.

[25] L. Chekhov, B. Eynard, N. Orantin, JHEP, 12 (2006), 053, 31 pp., arXiv: math-ph/0603003.

[26] A. Alexandrov, A. Mironov, A. Morozov, Internat. J. Modern Phys. A, 19:24 (2004), 4127-4165, arXiv: hep-th/0310113.

[27] B. Dubrovin, "Geometry of 2D topological field theories", Integrable Systems and Quantum groups (Montecatini Terme, Italy, June 14-22, 1993), Lecture Notes in Mathematics, 1620, eds. M. Francaviglia, S. Greco, Springer, Berlin, 1996, 120-348, arXiv: hep-th/9407018.

[28] P. Dunin-Barkowski, P. Norbury, N. Orantin, P. Popolitov, S. Shadrin, "Superpotentials and the Eynard-Orantin topological recursion", In preparation.

[29] J.E. Andersen, R. C. Penner, C. M. Reidys, M. S. Waterman, J. Math. Biol., 67:5 (2013), $1261-1278$.

[30] J. Harer, Invent. Math., 84:1 (1986), 157-176.

[31] K. Strebel, Quadratic Differentials, Ergebnisse der Mathematik und ihrer Grenzgebiete, 3, Springer, Berlin, 1984.

[32] R. C. Penner, Commun. Math. Phys., 113:2 (1987), 299-339.

[33] R. C. Penner, J. Differential Geom., 27:1 (1988), 35-53.

[34] M. L. Kontsevich, Commun. Math. Phys., 147:1 (1992), 1-23.

[35] S. Kharchev, A. Marshakov, A. Mironov, A. Morozov, A. Zabrodin, Phys. Lett. B, 275:3-4 (1992), 311-314, arXiv: hep-th/9111037.

[36] J. Ambjørn, L. Chekhov, Ann. Inst. Henri Poincaré D, 1:3 (2014), 337-361, arXiv: 1404.4240.

[37] A. Alexandrov, A. Mironov, A. Morozov, S. Natanzon, JHEP, 11 (2014), 080, 30 pp., arXiv: 1405.1395.

[38] J.E. Andersen, L. O. Chekhov, C. M. Reidys, R. C. Penner, P. Sułkowski, Nucl. Phys. B, 866:3 (2013), 414-443, arXiv: 1205.0658.

[39] S. Keel, Trans. Amer. Math. Soc., 330:2 (1992), 545-574.

[40] B. Dubrovin, "Geometry of 2D topological field theories", Integrable Systems and Quantum Groups (Montecatini Terme, Italy, June 14-22, 1993), Lecture Notes in Mathematics, 1620, eds. M. Francaviglia, S. Greco, Springer, Berlin, 1996, 120-348.

[41] C. Teleman, Invent. Math., 188:3 (2012), 525-588.

[42] U. Haagerup, S. Thorbjørnsen, Infin. Dimens. Anal. Quantum Probab. Relat. Top., 15:1 (2012), 1250003, 41 pp.

[43] R. J. Milgram, R. C. Penner, "Riemann's moduli space and the symmetric group", Mapping Class Groups and Moduli Spaces of Riemann Surfaces (Göttingen, Germany, June 24-28, 1991; Seattle, WA, USA, August 6-10, 1991), Contemporary Mathematics, 150, eds. C.-F. Bödigheimer, R. M. Hain, AMS, Providence, RI, 1993, 247-290.

[44] B. Fang, C.-C. M. Liu, Z. Zong, The Eynard-Orantin recursion and equivariant mirror symmetry for the projective line, arXiv: 1411.3557. 